Article

\title{
Some Interval-Valued Intuitionistic Fuzzy Dombi Hamy Mean Operators and Their Application for Evaluating the Elderly Tourism Service Quality in Tourism Destination
}

\author{
Liangping $\mathrm{Wu}^{1}{ }^{1}$, Guiwu Wei ${ }^{1}$, Hui Gao ${ }^{1, *}$ and Yu Wei ${ }^{2, *}$ \\ 1 School of Business, Sichuan Normal University, Chengdu 610101, China; wuliangping6@sicnu.edu.cn (L.W.); \\ weiguiwu@uestc.edu.cn (G.W.) \\ 2 School of Finance, Yunnan University of Finance and Economics, Kunming 650221, China \\ * Correspondence: gaohuisxy@sicnu.edu.cn (H.G.); weiyusy0@126.com (Y.W.)
}

Received: 29 September 2018; Accepted: 24 November 2018; Published: 1 December 2018

\begin{abstract}
In this paper, we expand the Hamy mean (HM) operator and Dombi operations with interval-valued intuitionistic fuzzy numbers (IVIFNs) to propose the interval-valued intuitionistic fuzzy Dombi Hamy mean (IVIFDHM) operator, interval-valued intuitionistic fuzzy weighted Dombi Hamy mean (IVIFWDHM) operator, interval-valued intuitionistic fuzzy dual Dombi Hamy mean (IVIFDDHM) operator, and interval-valued intuitionistic fuzzy weighted dual Dombi Hamy mean (IVIFWDDHM) operator. Then the MADM models are designed with IVIFWDHM and IVIFWDDHM operators. Finally, we gave an example for evaluating the elderly tourism service quality in tourism destination to show the proposed models.
\end{abstract}

Keywords: multiple attribute decision making (MADM); interval-valued intuitionistic fuzzy numbers (IVIFNs); interval-valued intuitionistic fuzzy weighted Dombi Hamy mean (IVIFWDHM) operator; interval-valued intuitionistic fuzzy weighted dual Dombi Hamy mean (IVIFWDDHM) operator; elderly tourism service quality; tourism destination

\section{Introduction}

The concept of intuitionistic fuzzy sets (IFSs) [1,2] has been utilized to deal with uncertainty and imprecision. Atanassov and Gargov [3] defined the interval-valued intuitionistic fuzzy sets (IVIFSs). $\mathrm{Xu}$ [4] introduced a method for the comparison between two intuitionistic fuzzy numbers (IFNs) and then develop some arithmetic aggregation operators. $\mathrm{Xu}$ and Yager [5] proposed some new geometric aggregation operators with IFNs. $\mathrm{Xu}$ and Chen [6] developed some interval-valued intuitionistic fuzzy geometric operators with interval-valued intuitionistic fuzzy numbers (IVIFNs). Wei [7] proposed two new aggregation operators: the induced intuitionistic fuzzy ordered weighted geometric (I-IFOWG) operator and the induced interval-valued intuitionistic fuzzy ordered weighted geometric (I-IIFOWG) operator. Wei [8] developed the gray relational analysis (GRA) for interval-valued intuitionistic fuzzy MADM with incompletely known attribute weight information. $\mathrm{Xu}$ and Chen [9] defined the Bonferroni mean for aggregating the IVIFNs based on the Bonferroni mean [10-17]. Chen [18] proposed the LINMAP (Linear Programming Technique for Multidimensional Analysis of Preference) model for MADM with IVIFNs. Hashemi, et al. [19] defined the multiple attribute group decision-making (MAGDM) model on the basis of the compromise ratio method with IVIFNs. Liu, et al. [20] gave the principal component analysis (IVIF-PCA) model for IVIFNs. Chen [21] proposed the interval-valued intuitionistic fuzzy preference ranking organization method for enrichment evaluations (IVIF-PROMETHEE) to deal with MADM. Dugenci [22] introduced a novel generalized distance 
measure for IVIFNs and illustrated the applicability of the proposed distance measure to MAGDM. Garg [23] defined a new generalized improved score function for IVIFNs. Until now, more and more decision making theories of IFSs and IVIFSs are extended to picture fuzzy set [24-33] and Pythagorean fuzzy sets [34-44].

Although IFSs and IVIFSs have been effectively used in some areas, all the existed approaches are unsuitable to depict the interrelationships among any number of IVIFNs assigned by a variable vector. The Hamy mean (HM) operator [38,45-48] and dual Hamy mean (DHM) operator [49] are famous operators which can show interrelationships among any number of arguments assigned by a variable vector. Therefore, the HM and DHM operators can assign a robust and flexible mechanism to solve the information fusion in MADM problems. Thus, we propose some HM operator to overcome this limits. Thus, how to aggregate these IVIFNs-based the traditional HM operators based on the Dombi operations [50-53] is an interesting issue. So, the purpose of this paper is to propose some HM and DHM operators to solve the MADM for evaluating the elderly tourism service quality in tourism destination with IVIFNs. In order to do so, the rest of this paper is organized as follows. In Section 2, we introduce the IVIFNs. In Section 3, we develop some HM operators with IVIFNs based on the Dombi operations. In Section 4, we present an example for evaluating the elderly tourism service quality in tourism destination with IVIFNs. Section 5 ends this paper with some comments.

\section{Preliminaries}

\subsection{IFSs and IVIFSs}

The concept of IFSs and IVIFSs are introduced.

Definition 1 [1,2]. An IFS $Q$ in $X$ is designed by

$$
Q=\left\{\left\langle x, \theta_{Q}(x), \vartheta_{Q}(x)\right\rangle \mid x \in X\right\}
$$

where $\theta_{Q}: X \rightarrow[0,1]$ and $\vartheta_{Q}: X \rightarrow[0,1]$, and $0 \leq \theta_{Q}(x)+\vartheta_{Q}(x) \leq 1, \forall x \in X$. The number $\theta_{Q}(x)$ and $\vartheta_{Q}(x)$ represents, respectively, the membership degree and non-membership degree of the element $x$ to the set $Q$.

Definition 2 [3]. Let X be an universe of discourse, An IVIFS $\widetilde{Q}$ over X is an object having the form as follows:

$$
\widetilde{Q}=\left\{\left\langle x, \widetilde{\theta}_{\widetilde{Q}}(x), \widetilde{\vartheta}_{\widetilde{Q}}(x)\right\rangle \mid x \in X\right\}
$$

where $\widetilde{\theta}_{\widetilde{Q}}(x) \subseteq[0,1]$ and $\widetilde{\vartheta}_{\widetilde{Q}}(x) \subseteq[0,1]$ are interval numbers, and $0 \leq \sup \left(\widetilde{\theta}_{\widetilde{Q}}(x)\right)+\sup \left(\widetilde{\vartheta}_{\widetilde{Q}}(x)\right) \leq 1$, $\forall x \in X$. For convenience, let $\widetilde{\theta}_{\widetilde{Q}}(x)=[e, f], \widetilde{\vartheta}_{\widetilde{Q}}(x)=[g, h]$, so $\widetilde{\varphi}=([e, f],[g, h])$ is an IVIFNs.

Definition 3 [54]. Let $\widetilde{\varphi}=([e, f],[g, h])$ be an IVIFN, a score function $S$ can be defined as follows:

$$
S(\widetilde{\varphi})=\frac{e-g+f-h}{2}, S(\widetilde{\varphi}) \in[-1,1]
$$

Definition 4 [54]. Let $\widetilde{\varphi}=([e, f],[g, h])$ be an IVIFN, an accuracy function $H$ can be defined as follows:

$$
H(\widetilde{\varphi})=\frac{e+f+g+h}{2}, H(\widetilde{\varphi}) \in[0,1] .
$$

To evaluate the degree of accuracy of the IVIFN $\widetilde{\varphi}=([e, f],[g, h])$.

Definition 5 [54]. Let $\widetilde{\varphi}_{1}=\left(\left[e_{1}, f_{1}\right],\left[g_{1}, h_{1}\right]\right)$ and $\widetilde{\varphi}_{2}=\left(\left[e_{2}, f_{2}\right],\left[g_{2}, h_{2}\right]\right)$ be two IVIFNs, s $\left(\widetilde{\varphi}_{1}\right)=$ $\frac{e_{1}-f_{1}+g_{1}-h_{1}}{2}$ and $s\left(\widetilde{\varphi}_{2}\right)=\frac{e_{2}-f_{2}+g_{2}-h_{2}}{2}$ be the scores of $\widetilde{\varphi}_{1}$ and $\widetilde{\varphi}_{2}$, respectively, and let $H\left(\widetilde{\varphi}_{1}\right)=\frac{e_{1}+f_{1}+g_{1}+h_{1}}{2}$ 
and $H\left(\widetilde{\varphi}_{2}\right)=\frac{e_{2}+f_{2}+g_{2}+h_{2}}{2}$ be the accuracy degrees of $\widetilde{\varphi}_{1}$ and $\widetilde{\varphi}_{2}$, respectively, then if $S\left(\widetilde{\varphi}_{1}\right)<S\left(\widetilde{\varphi}_{2}\right)$, then $\widetilde{\varphi}_{1}<\widetilde{\varphi}_{2} ;$ if $S\left(\widetilde{\varphi}_{1}\right)=S\left(\widetilde{\varphi}_{2}\right)$, then (1) if $H\left(\widetilde{\varphi}_{1}\right)=H\left(\widetilde{\varphi}_{2}\right)$, then $\widetilde{\varphi}_{1}=\widetilde{\varphi}_{2} ;$ (2) if $H\left(\widetilde{\varphi}_{1}\right)<H\left(\widetilde{\varphi}_{2}\right)$, then $\widetilde{\varphi}_{1}<\widetilde{\varphi}_{2}$.

Definition 6 [54]. For two IVIFNs $\widetilde{\varphi}_{1}=\left(\left[e_{1}, f_{1}\right],\left[g_{1}, h_{1}\right]\right)$ and $\widetilde{\varphi}_{2}=\left(\left[e_{2}, f_{2}\right],\left[g_{2}, h_{2}\right]\right)$, the following operational laws are defined as follows:

(1) $\widetilde{\varphi}_{1} \oplus \widetilde{\varphi}_{2}=\left(\left[e_{1}+e_{2}-e_{1} e_{2}, f_{1}+f_{2}-f_{1} f_{2}\right],\left[g_{1} g_{2}, h_{1} h_{2}\right]\right)$;

(2) $\widetilde{\varphi}_{1} \otimes \widetilde{\varphi}_{2}=\left(\left[e_{1} e_{2}, f_{1} f_{2}\right],\left[g_{1}+g_{2}-g_{1} g_{2}, h_{1}+h_{2}-h_{1} h_{2}\right]\right)$;

(3) $\lambda \widetilde{\varphi}_{1}=\left(\left[1-\left(1-e_{1}\right)^{\lambda}, 1-\left(1-f_{1}\right)^{\lambda}\right],\left[g_{1}^{\lambda}, h_{1}^{\lambda}\right]\right), \lambda>0$;

(4) $\left(\widetilde{\varphi}_{1}\right)^{\lambda}=\left(\left[e_{1}^{\lambda}, f_{1}^{\lambda}\right],\left[1-\left(1-g_{1}\right)^{\lambda}, 1-\left(1-h_{1}\right)^{\lambda}\right]\right), \lambda>0$.

\subsection{HM Operator}

Hara, Uchiyama and Takahasi [48] proposed the HM operator.

Definition 7 [48]. The HM operator is defined as follows:

$$
\operatorname{HM}^{(x)}\left(\varphi_{1}, \varphi_{2}, \cdots, \varphi_{n}\right)=\frac{\sum_{1 \leq i_{1}<\ldots<i_{x} \leq n}\left(\prod_{j=1}^{x} \varphi_{i_{j}}\right)^{\frac{1}{x}}}{C_{n}^{x}}
$$

where $x$ is a parameter, $x=1,2, \ldots, n, i_{1}, i_{2}, \ldots, i_{x}$ are $x$ integer values taken from the set $\{1,2, \ldots, n\}$ of $k$ integer values, $C_{n}^{x}$ is the binomial coefficient, $C_{n}^{x}=\frac{n !}{x !(n-x) !}$.

\subsection{Dombi Operations of IVIFNs}

Definition 8 [50]. Dombi [50] proposed a generator to produce Dombi T-norm and T-conorm which are shown as follows:

$$
\begin{gathered}
\mathrm{D}(q, r)=\frac{1}{1+\left(\left(\frac{1-q}{q}\right)^{\beta}+\left(\frac{1-r}{r}\right)^{\beta}\right)^{1 / \beta}} \\
\mathrm{D}^{c}(q, r)=1-\frac{1}{1+\left(\left(\frac{q}{1-q}\right)^{\beta}+\left(\frac{r}{1-r}\right)^{\beta}\right)^{1 / \beta}}
\end{gathered}
$$

where $\beta>0,(q, r) \in[0,1]$.

Based on the Dombi T-norm and T-conorm, we can give the operational rules of IVIFNs.

Definition 9. For two IVIFNs $\widetilde{\varphi}_{1}=\left(\left[e_{1}, f_{1}\right],\left[g_{1}, h_{1}\right]\right)$ and $\widetilde{\varphi}_{2}=\left(\left[e_{2}, f_{2}\right],\left[g_{2}, h_{2}\right]\right), \lambda>0$, the Dombi operational laws are defined as follows:

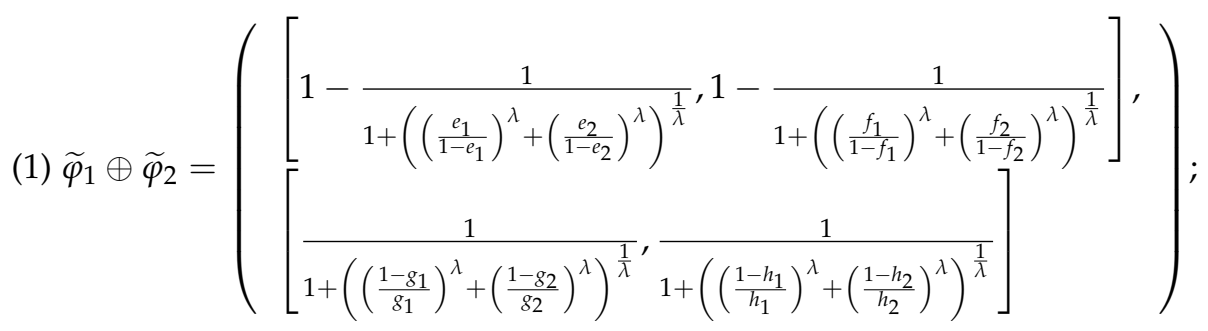


(2) $\widetilde{\varphi}_{1} \otimes \widetilde{\varphi}_{2}=\left(\begin{array}{l}{\left[\frac{1}{1+\left(\left(\frac{1-e_{1}}{e_{1}}\right)^{\lambda}+\left(\frac{1-e_{2}}{e_{2}}\right)^{\lambda}\right)^{\frac{1}{\lambda}}}, \frac{1}{1+\left(\left(\frac{1-f_{1}}{f_{1}}\right)^{\lambda}+\left(\frac{1-f_{2}}{f_{2}}\right)^{\lambda}\right)^{\frac{1}{\lambda}}}\right]} \\ {\left[1-\frac{1}{1+\left(\left(\frac{g_{1}}{1-g_{1}}\right)^{\lambda}+\left(\frac{g_{2}}{1-g_{2}}\right)^{\lambda}\right)^{\frac{1}{\lambda}}, 1-\frac{1}{1+\left(\left(\frac{h_{1}}{1-h_{1}}\right)^{\lambda}+\left(\frac{h_{2}}{1-h_{2}}\right)^{\lambda}\right)^{\frac{1}{\lambda}}}}\right]}\end{array}\right)$;

(3) $n \widetilde{\varphi}_{1}=\left(\left[1-\frac{1}{1+\left(n\left(\frac{e_{1}}{1-e_{1}}\right)^{\lambda}\right)^{\frac{1}{\lambda}}}, 1-\frac{1}{1+\left(n\left(\frac{f_{1}}{1-f_{1}}\right)^{\lambda}\right)^{\frac{1}{\lambda}}}\right],\left[\frac{1}{1+\left(n\left(\frac{1-g_{1}}{g_{1}}\right)^{\lambda}\right)^{\frac{1}{\lambda}}}, \frac{1}{1+\left(n\left(\frac{1-h_{1}}{h_{1}}\right)^{\lambda}\right)^{\frac{1}{\lambda}}}\right]\right)$;

(4) $\left(\widetilde{\varphi}_{1}\right)^{n}=\left(\left[\frac{1}{1+\left(n\left(\frac{1-e_{1}}{e_{1}}\right)^{\lambda}\right)^{\frac{1}{\lambda}}}, \frac{1}{1+\left(n\left(\frac{1-f_{1}}{f_{1}}\right)^{\lambda}\right)^{\frac{1}{\lambda}}}\right],\left[1-\frac{1}{1+\left(n\left(\frac{g_{1}}{1-g_{1}}\right)^{\lambda}\right)^{\frac{1}{\lambda}}}, 1-\frac{1}{1+\left(n\left(\frac{h_{1}}{1-h_{1}}\right)^{\lambda}\right)^{\frac{1}{\lambda}}}\right]\right)$.

\section{Some Dombi Hamy Mean Operators with IVIFNs}

\subsection{The IVIFDHM Operator}

Based on the HM operator and Dombi operation rules, the IVIFDHM operator is defined as follows:

Definition 10. Let $\widetilde{\varphi}_{j}=\left(\left[e_{j}, f_{j}\right],\left[g_{j}, h_{j}\right]\right)(j=1,2, \ldots, n)$ be a set of IVIFNs. The IVIFDHM operator is

$$
\operatorname{IVIFDHM}^{(x)}\left(\widetilde{\varphi}_{1}, \widetilde{\varphi}_{2}, \ldots, \widetilde{\varphi}_{n}\right)=\frac{\underset{1 \leq i_{1}<\ldots<i_{x} \leq n}{\oplus}\left(\stackrel{x}{\otimes} \widetilde{\varphi}_{j=1} \widetilde{\varphi}_{j}\right)^{\frac{1}{x}}}{C_{n}^{x}}
$$

Theorem 1. Let $\widetilde{\varphi}_{j}=\left(\left[e_{j}, f_{j}\right],\left[g_{j}, h_{j}\right]\right)(j=1,2, \ldots, n)$ be a set of IVIFNs. The fused value by the IVIFDHM operators is also an IVIFN where

$$
\begin{aligned}
& \operatorname{IVIFDHM}^{(x)}\left(\widetilde{\varphi}_{1}, \widetilde{\varphi}_{2}, \ldots, \widetilde{\varphi}_{n}\right)=\frac{\underset{1 \leq i_{1}<\ldots<i x \leq n}{\oplus}\left(\underset{\substack{x \\
\mathcal{N}_{j=1}}}{\widetilde{\varphi}_{i_{j}}}\right)^{\frac{1}{x}}}{C_{n}^{x}}
\end{aligned}
$$

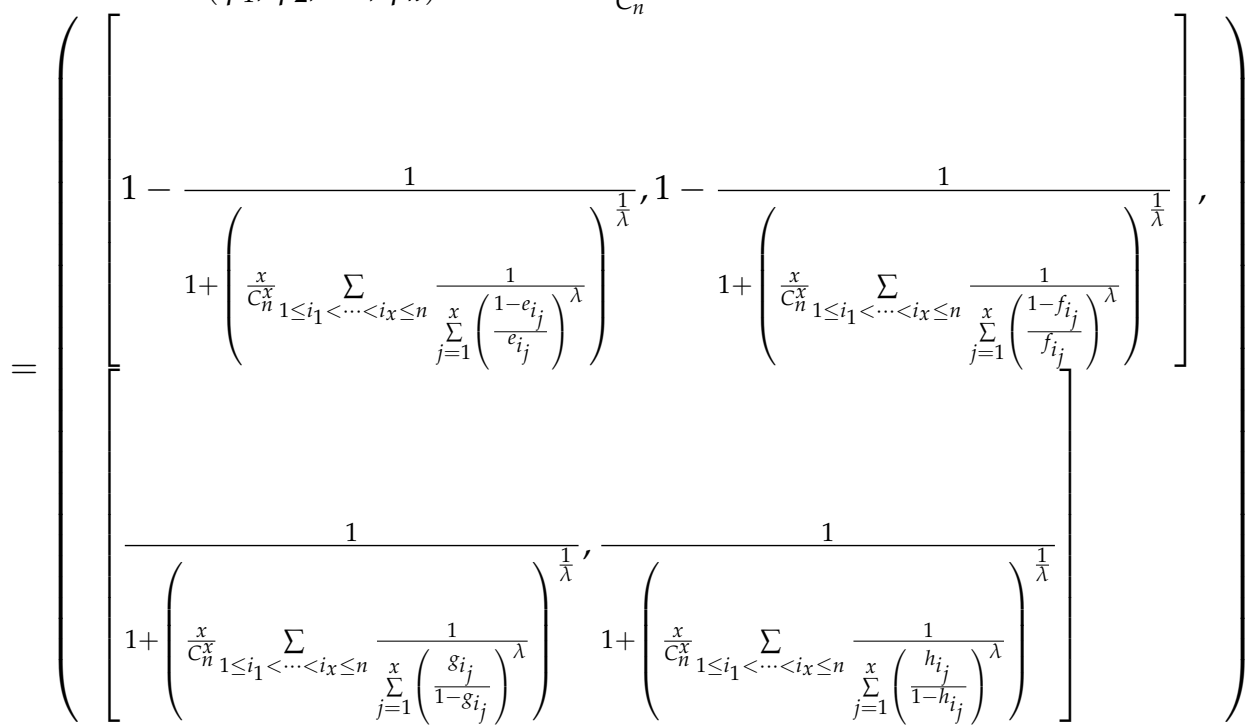




\section{Proof.}

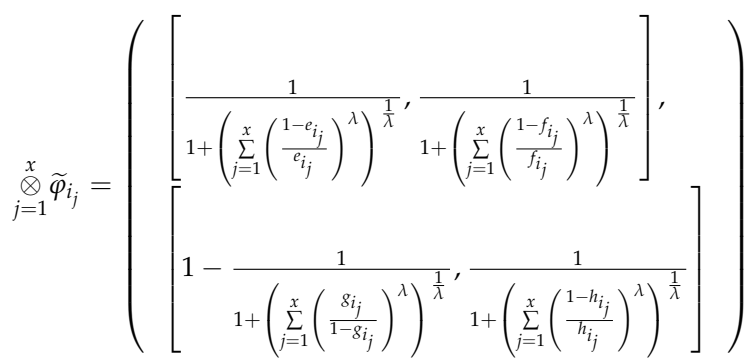

Thus,

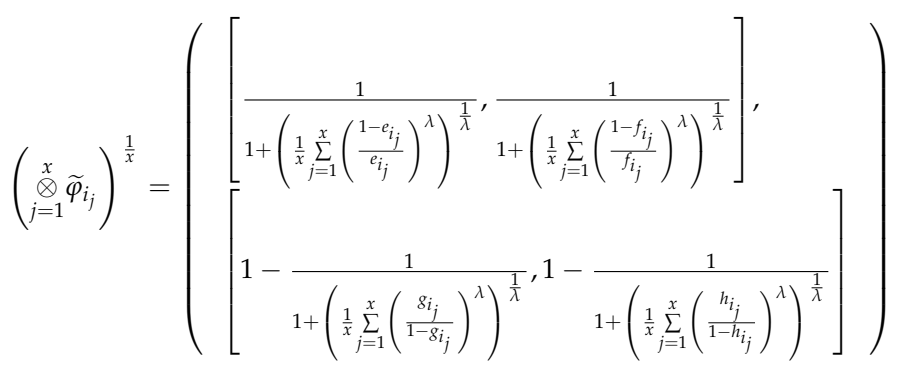

Thereafter,

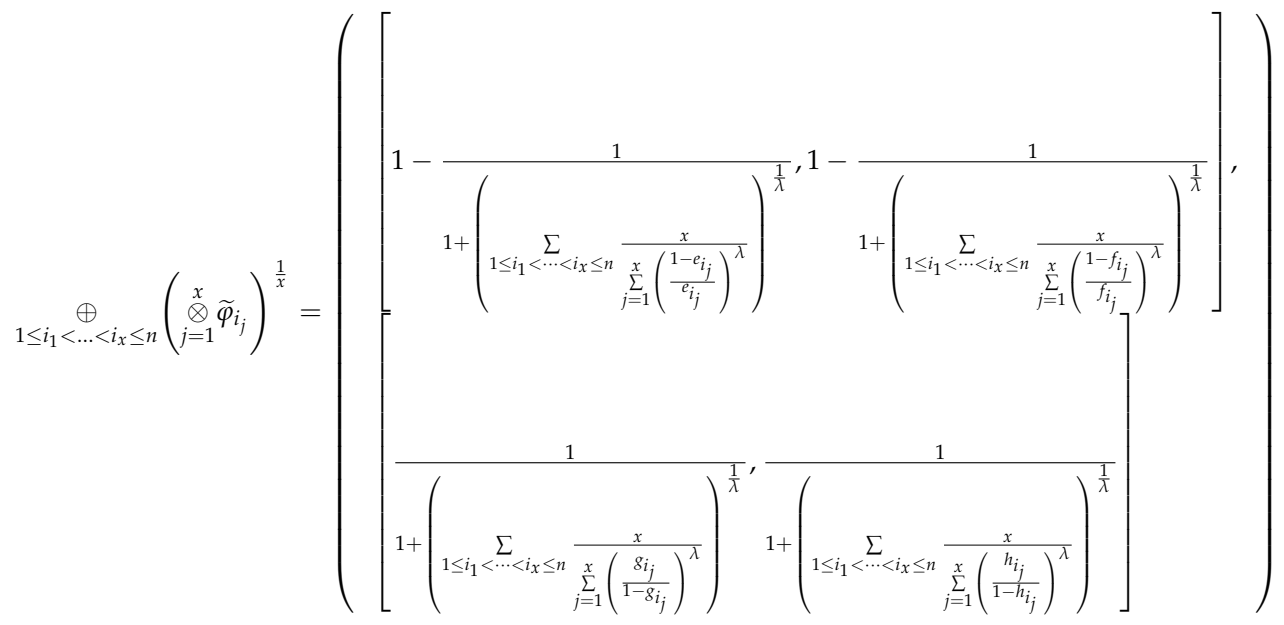

Therefore,

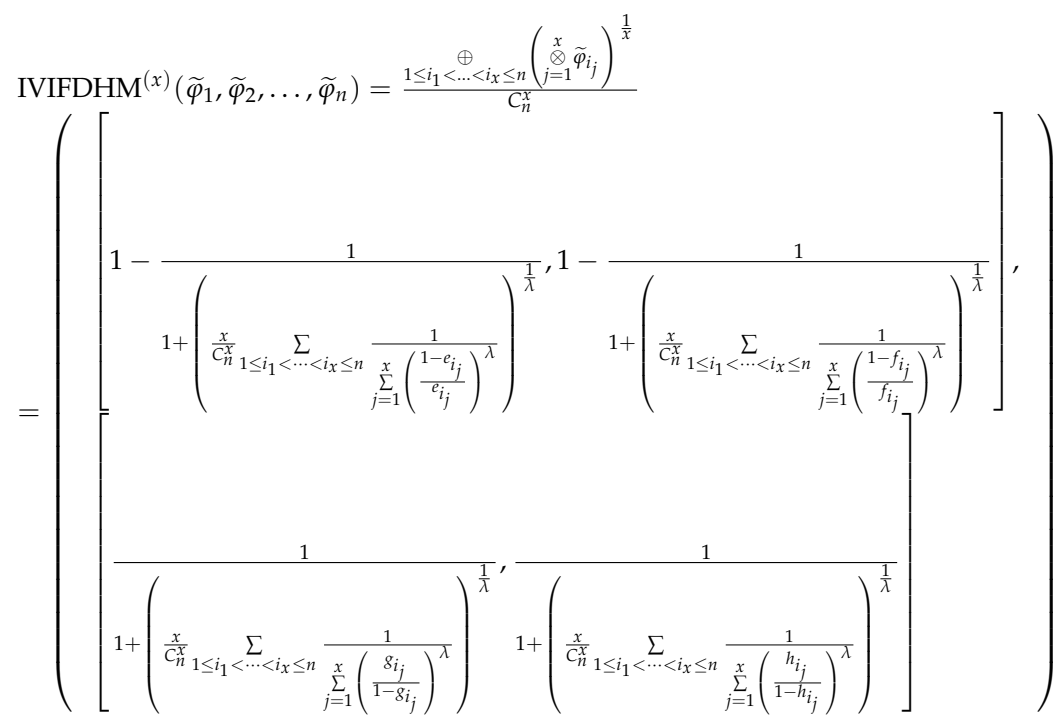

Thus Equation (9) is right. $\square$ 
Example 1. Let $\widetilde{\varphi}_{1}=([0.2,0.4],[0.3,0.6]), \widetilde{\varphi}_{2}=([0.1,0.3],[0.2,0.5]), \widetilde{\varphi}_{3}=([0.3,0.5],[0.1,0.2])$ and $\widetilde{\varphi}_{4}=([0.1,0.4],[0.3,0.5])$ be four IVIFNs, and $x=2, \lambda=3$,

$$
\begin{gathered}
\frac{1-e_{i_{j}}}{e_{i_{j}}}=(4.0000,9.0000,2.3333,9.0000), \frac{1-f_{i_{j}}}{f_{i_{j}}}=(1.5000,2.3333,1.0000,1.5000) \\
\frac{g_{i_{j}}}{1-g_{i_{j}}}=(0.4286,0.2500,0.1111,0.4286), \frac{h_{i_{j}}}{1-h_{i_{j}}}=(1.5000,1.0000,0.2500,1.0000)
\end{gathered}
$$

Then according to Equation (9), we have

$$
\begin{aligned}
& \operatorname{IFDHM}^{(x)}\left(\widetilde{\varphi}_{1}, \widetilde{\varphi}_{2}, \ldots, \widetilde{\varphi}_{n}\right)=\frac{\left.\underset{1 \leq i_{1}<\ldots<i_{x} \leq n}{\stackrel{\oplus}{\ominus}\left(\underset{x}{\otimes} \widetilde{\varphi}_{i_{j}}\right.}\right)^{\frac{1}{x}}}{C_{n}^{x}}
\end{aligned}
$$

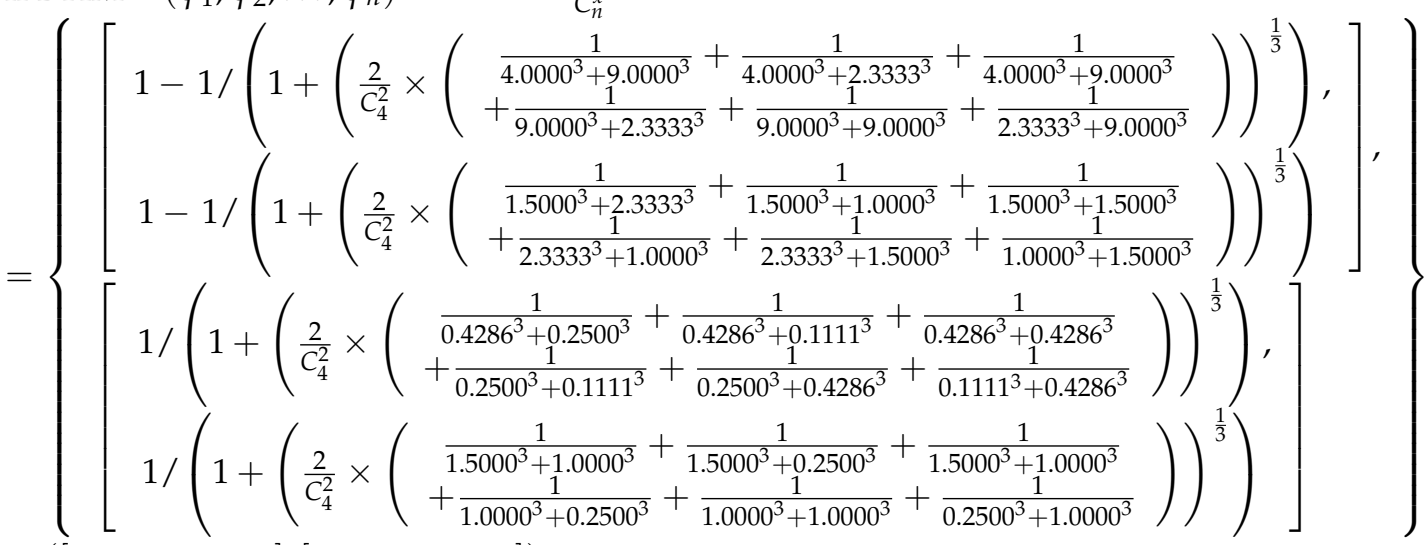

$$
\begin{aligned}
& =([0.1560,0.3919],[0.2306,0.4941])
\end{aligned}
$$

Then we list some properties of IVIFDHM operator.

Property 1. (Idempotency) If $\widetilde{\varphi}_{j}=\left(\left[e_{j}, f_{j}\right],\left[g_{j}, h_{j}\right]\right)(j=1,2, \ldots, n)=\widetilde{\varphi}$ are equal, then

$$
\operatorname{IVIFDHM}^{(x)}\left(\widetilde{\varphi}_{1}, \widetilde{\varphi}_{2}, \ldots, \widetilde{\varphi}_{n}\right)=\widetilde{\varphi}
$$

Property 2. (Monotonicity) Let $\widetilde{\varphi}_{j}=\left(\left[e_{j}, f_{j}\right],\left[g_{j}, h_{j}\right]\right)(j=1,2, \ldots, n)$ and $\widetilde{\theta}_{j}=\left(\left[r_{j}, s_{j}\right],\left[m_{j}, n_{j}\right]\right)(j=$ $1,2, \ldots, n)$ be two sets of IVIFNs. If $e_{j} \leq r_{j}, f_{j} \leq s_{j}$ and $g_{j} \geq m_{j}, h_{j} \geq n_{j}$ hold for all $j$, then

$$
\operatorname{IVIFDHM}^{(x)}\left(\widetilde{\varphi}_{1}, \widetilde{\varphi}_{2}, \ldots, \widetilde{\varphi}_{n}\right) \leq \operatorname{IVIFDHM}^{(x)}\left(\widetilde{\theta}_{1}, \widetilde{\theta}_{2}, \ldots, \widetilde{\theta}_{n}\right)
$$

Property 3. (Boundedness) Let $\widetilde{\varphi}_{j}=\left(\left[e_{j}, f_{j}\right],\left[g_{j}, h_{j}\right]\right)(j=1,2, \ldots, n)$ be a set of IVIFNs. If $\widetilde{\varphi}_{i}^{+}=\left(\left(\left[\max _{i}\left(e_{j}\right), \max _{i}\left(f_{j}\right)\right],\left[\min _{i}\left(g_{j}\right), \min _{i}\left(h_{j}\right)\right]\right)\right)$ and $\widetilde{\varphi}_{i}^{-}=\left(\left[\min _{i}\left(e_{j}\right), \min _{i}\left(f_{j}\right)\right]\right.$, $\left.\left[\max _{i}\left(g_{j}\right), \max _{i}\left(h_{j}\right)\right]\right)$ then

$$
\widetilde{\varphi}^{-} \leq \operatorname{IVIFDHM}^{(x)}\left(\widetilde{\varphi}_{1}, \widetilde{\varphi}_{2}, \ldots, \widetilde{\varphi}_{n}\right) \leq \widetilde{\varphi}^{+}
$$

\subsection{The IVIFWDHM Operator}

In real MADM, it is important to pay attention to attribute weights. Thus we propose the interval-valued intuitionistic fuzzy weighted Dombi Hamy mean (IVIFWDHM) operator. 
Definition 11. Let $\widetilde{\varphi}_{j}=\left(\left[e_{j}, f_{j}\right],\left[g_{j}, h_{j}\right]\right)(j=1,2, \ldots, n)$ be a set of IVIFNs with their weight vector $w_{i}=\left(w_{1}, w_{2}, \ldots, w_{n}\right)^{T}$, thereby satisfying $w_{i} \in[0,1]$ and $\sum_{i=1}^{n} w_{i}=1$. Then the IVIFWDHM operator is as follows:

$$
\operatorname{IVIFWDHM}_{w}^{(x)}\left(\varphi_{1}, \varphi_{2}, \ldots, \varphi_{n}\right)=\frac{\underset{1 \leq i_{1}<\ldots<i_{x} \leq n}{\oplus}\left(\stackrel{x}{\otimes}\left(\widetilde{\varphi}_{i_{j}}\right)^{w_{i_{j}}}\right)^{\frac{1}{x}}}{C_{n}^{x}}
$$

Theorem 2. Let $\widetilde{\varphi}_{j}=\left(\left[e_{j}, f_{j}\right],\left[g_{j}, h_{j}\right]\right)(j=1,2, \ldots, n)$ be a set of IVIFNs. The fused value by IVIFWDHM operators is also an IVIFN where

$$
\begin{aligned}
& \operatorname{IVIFWDHM}_{w}^{(x)}\left(\widetilde{\varphi}_{1}, \widetilde{\varphi}_{2}, \ldots, \widetilde{\varphi}_{n}\right)=\frac{\underset{1 \leq i_{1}<\ldots<i_{x} \leq n}{\oplus}\left({ }_{j=1}^{x}\left(\widetilde{\varphi}_{i_{j}}\right)^{w_{i_{j}}}\right)^{\frac{1}{x}}}{C_{n}^{x}}
\end{aligned}
$$

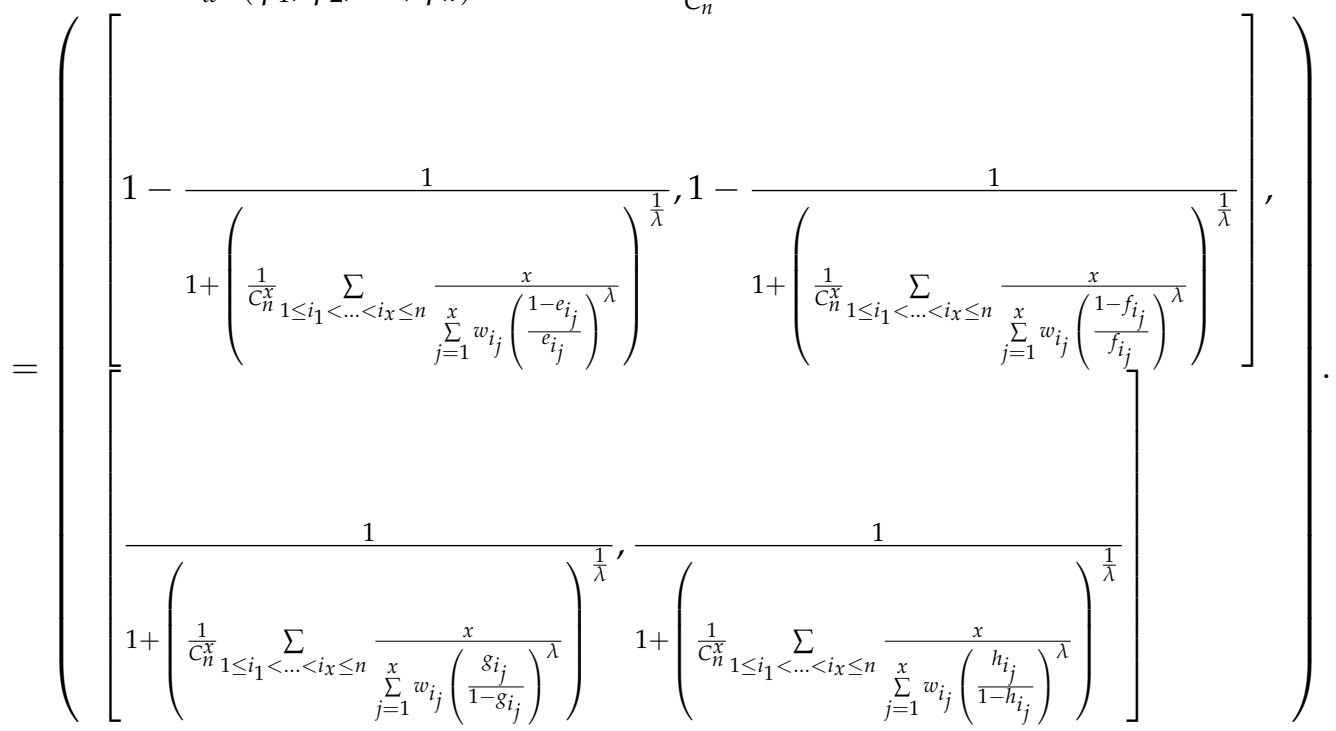

\section{Proof.}

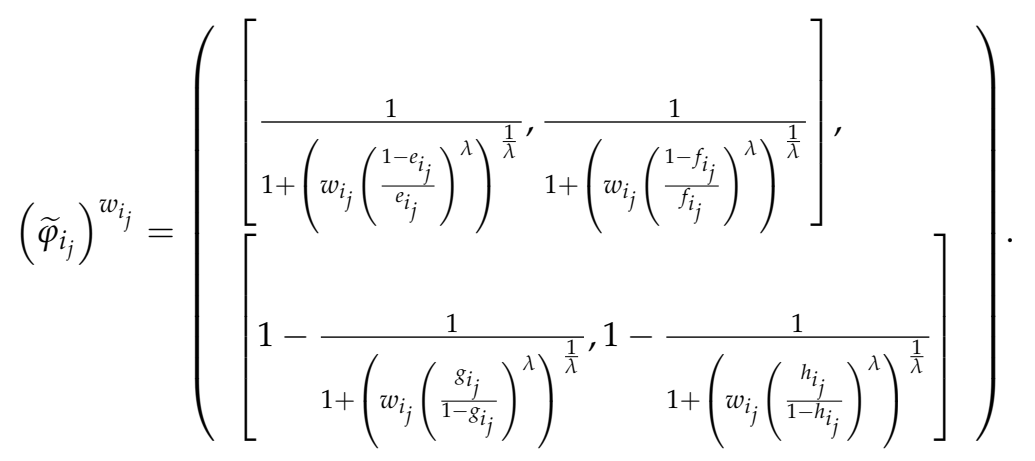

Thus,

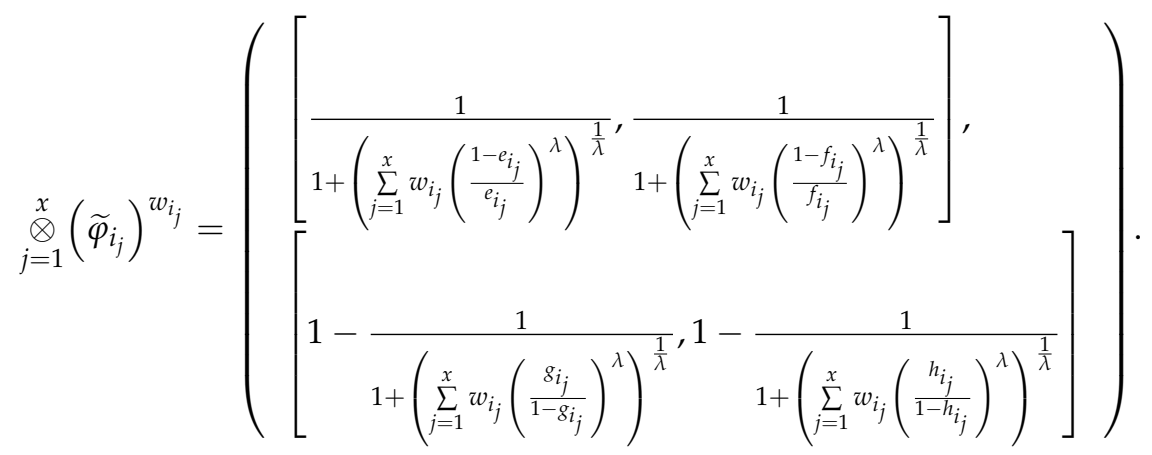


Therefore,

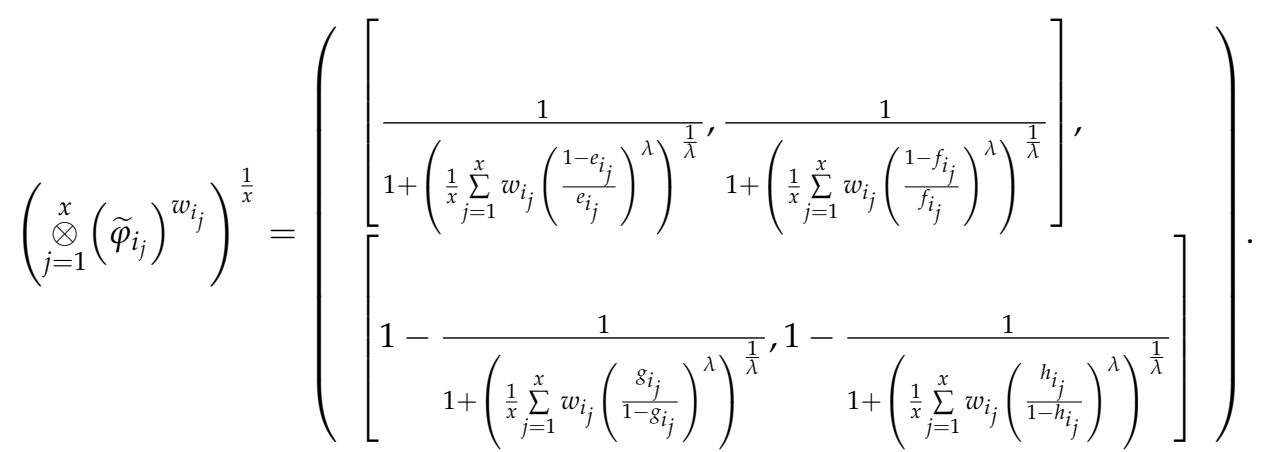

Thereafter,

$$
\begin{aligned}
& \underset{1 \leq i_{1}<\ldots<i_{x} \leq n}{\oplus}\left(\stackrel{x}{\stackrel{x}{\otimes}}\left(\widetilde{\varphi}_{i_{j}}\right)^{w_{i_{j}}}\right)^{\frac{1}{x}}
\end{aligned}
$$

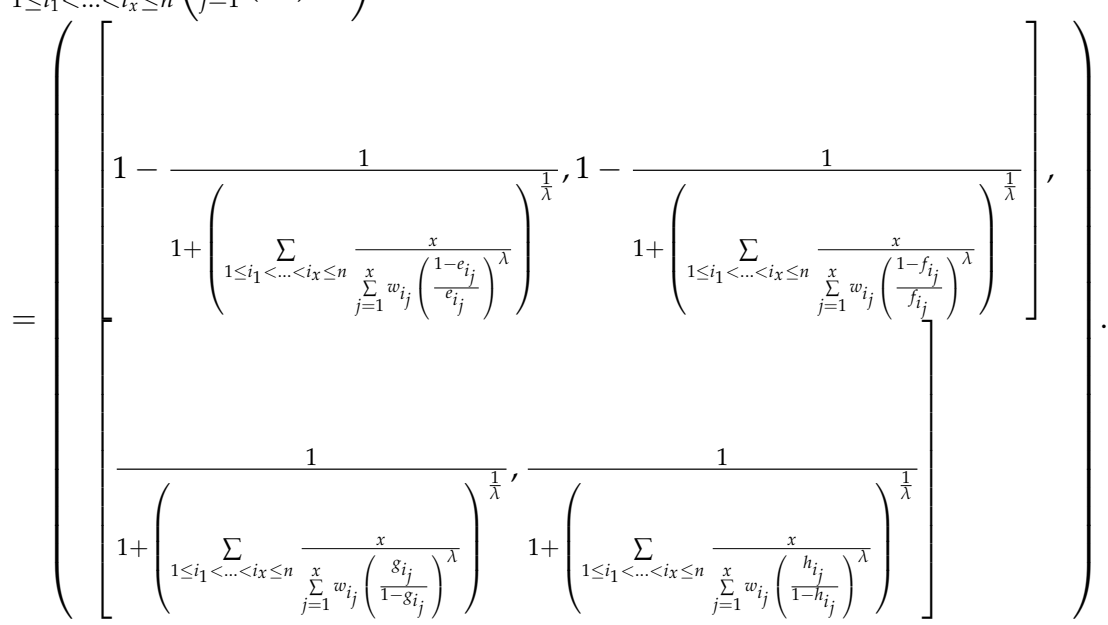

Therefore,

$$
\begin{aligned}
& \operatorname{IVIFWDHM}_{w}^{(x)}\left(\widetilde{\varphi}_{1}, \widetilde{\varphi}_{2}, \ldots, \widetilde{\varphi}_{n}\right)=\frac{\underset{1 \leq i_{1}<\ldots<i x \leq n}{\oplus}\left(\stackrel{\bigotimes}{j=1}_{j}^{x}\left(\widetilde{\varphi}_{i_{j}}\right)^{w_{i j}}\right)^{\frac{1}{x}}}{C_{n}^{x}}
\end{aligned}
$$

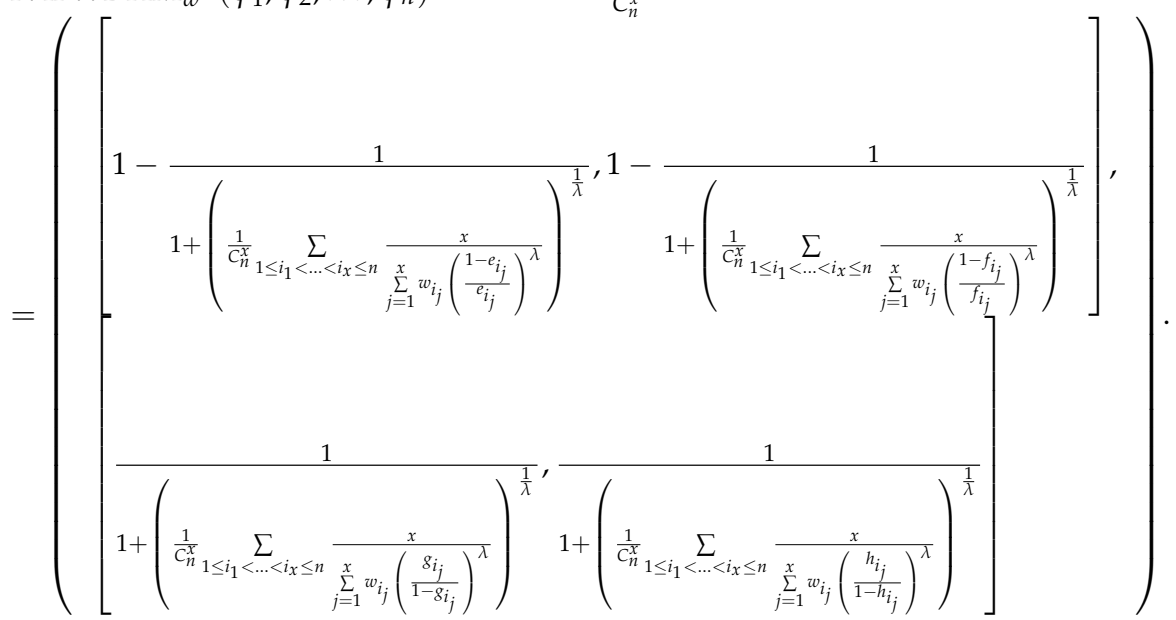

Hence, Equation (18) is kept. $\square$ 
Example 2. Let $\widetilde{\varphi}_{1}=([0.2,0.4],[0.3,0.6]), \widetilde{\varphi}_{2}=([0.1,0.3],[0.2,0.5]), \widetilde{\varphi}_{3}=([0.3,0.5],[0.1,0.2])$ and $\widetilde{\varphi}_{4}=([0.1,0.4],[0.3,0.5])$ be four IVIFNs, and $x=2, \lambda=3, w=(0.4,0.1,0.3,0.2)$,

$$
\begin{aligned}
\frac{1-e_{i_{j}}}{e_{i_{j}}}=(4.0000,9.0000,2.3333,9.0000), \frac{1-f_{i_{j}}}{f_{i_{j}}}=(1.5000,2.3333,1.0000,1.5000) \\
\frac{g_{i_{j}}}{1-g_{i_{j}}}=(0.4286,0.2500,0.1111,0.4286), \frac{h_{i_{j}}}{1-h_{i_{j}}}=(1.5000,1.0000,0.2500,1.0000)
\end{aligned}
$$

Then according to Equation (18), we have

$$
\begin{aligned}
& \operatorname{IVIFWDHM}_{w}^{(x)}\left(\widetilde{\varphi}_{1}, \widetilde{\varphi}_{2}, \ldots, \widetilde{\varphi}_{n}\right)=\frac{\underset{1 \leq i_{1}<\ldots<i_{x} \leq n}{\oplus}\left(\underset{\substack{x \\
j=1}}{\otimes}\left(\widetilde{\varphi}_{i_{j}}\right)^{w_{i_{j}}}\right)^{\frac{1}{x}}}{C_{n}^{x}}
\end{aligned}
$$

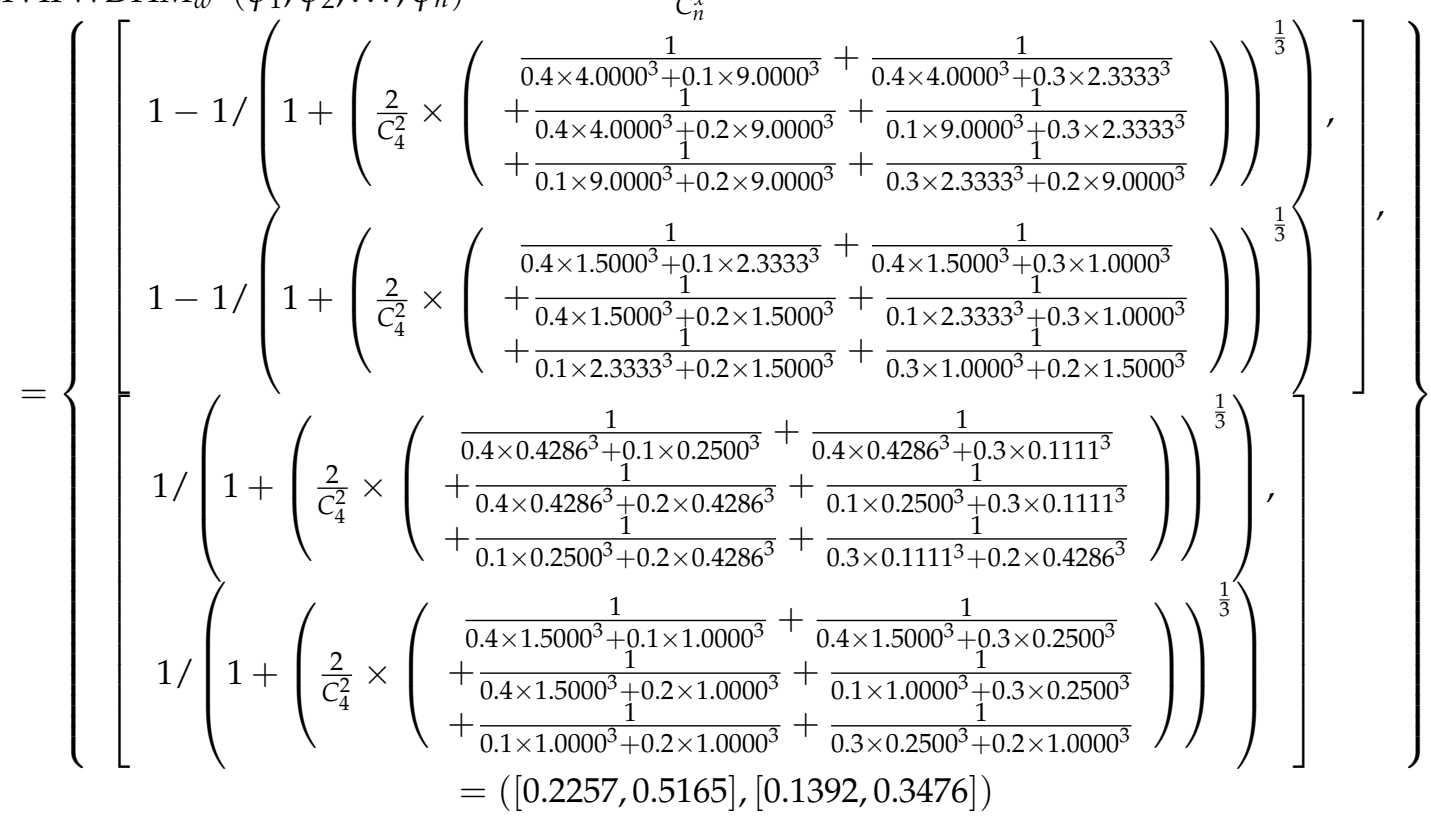

Then we list some properties of the IVIFWDHM operator.

Property 4. (Monotonicity) Let $\widetilde{\varphi}_{j}=\left(\left[e_{j}, f_{j}\right],\left[g_{j}, h_{j}\right]\right)(j=1,2, \ldots, n)$ and $\widetilde{\theta}_{j}=\left(\left[r_{j}, s_{j}\right],\left[m_{j}, n_{j}\right]\right)(j=$ $1,2, \ldots, n)$ be two sets of IVIFNs. If $e_{j} \leq r_{j}, f_{j} \leq s_{j}$ and $g_{j} \geq m_{j}, h_{j} \geq n_{j}$ hold for all $j$, then

$$
\operatorname{IVIFWDHM}^{(x)}\left(\widetilde{\varphi}_{1}, \widetilde{\varphi}_{2}, \ldots, \widetilde{\varphi}_{n}\right) \leq \operatorname{IVIFWDHM}^{(x)}\left(\widetilde{\theta}_{1}, \widetilde{\theta}_{2}, \ldots, \widetilde{\theta}_{n}\right)
$$

The proof is similar to IVIFDHM, thus, it is omitted here.

Property 5. (Boundedness) Let $\widetilde{\varphi}_{j}=\left(\left[e_{j}, f_{j}\right],\left[g_{j}, h_{j}\right]\right)(j=1,2, \ldots, n)$ be a set of IVIFNs. If $\widetilde{\varphi}_{i}^{+}=\left(\left(\left[\max _{i}\left(e_{j}\right), \max _{i}\left(f_{j}\right)\right],\left[\min _{i}\left(g_{j}\right), \min _{i}\left(h_{j}\right)\right]\right)\right)$ and $\widetilde{\varphi}_{i}^{-}=\left(\left[\min _{i}\left(e_{j}\right), \min _{i}\left(f_{j}\right)\right]\right.$, $\left.\left[\max _{i}\left(g_{j}\right), \max _{i}\left(h_{j}\right)\right]\right)$ then

$$
\widetilde{\varphi}^{-} \leq \operatorname{IVIFWDHM}^{(x)}\left(\widetilde{\varphi}_{1}, \widetilde{\varphi}_{2}, \ldots, \widetilde{\varphi}_{n}\right) \leq \widetilde{\varphi}^{+}
$$




\subsection{The IVIFDDHM Operator}

Wu, Wang, Wei, and Wei [49] proposed the dual HM (DHM) operator.

Definition 12 [49]. The DHM operator is as follows:

$$
\operatorname{DHM}^{(x)}\left(\varphi_{1}, \varphi_{2}, \cdots, \varphi_{n}\right)=\left(\prod_{1 \leq i_{1}<\ldots<i_{x} \leq n}\left(\frac{\sum_{j=1}^{x} \varphi_{i_{j}}}{x}\right)\right)^{\frac{1}{\mathcal{C}_{n}^{x}}}
$$

where $x$ is a parameter and $x=1,2, \ldots, n, i_{1}, i_{2}, \ldots, i_{x}$ are $x$ integer values taken from the set $\{1,2, \ldots, n\}$ of $k$ integer values, $C_{n}^{x}$ denotes the binomial coefficient and $C_{n}^{x}=\frac{n !}{x !(n-x) !}$.

In this section, we will propose the interval-valued intuitionistic fuzzy Dombi DHM (IVIFDDHM) operator.

Definition 13. Let $\widetilde{\varphi}_{j}=\left(\left[e_{j}, f_{j}\right],\left[g_{j}, h_{j}\right]\right)(j=1,2, \ldots, n)$ be a set of IVIFNs. The IVIFDDHM operator is as follows:

$$
\operatorname{IVIFDDHM}^{(x)}\left(\widetilde{\varphi}_{1}, \widetilde{\varphi}_{2}, \cdots, \widetilde{\varphi}_{n}\right)=\left(\underset{1 \leq i_{1}<\ldots<i_{x} \leq n}{\otimes}\left(\frac{\underset{j=1}{\oplus} \widetilde{\varphi}_{i_{j}}}{x}\right)\right)^{\frac{1}{\mathcal{C}_{n}^{x}}}
$$

Theorem 3. Let $\widetilde{\varphi}_{j}=\left(\left[e_{j}, f_{j}\right],\left[g_{j}, h_{j}\right]\right)(j=1,2, \ldots, n)$ be a set of IVIFNs. The fused value by IVIFDDHM operators is also an IVIFN where

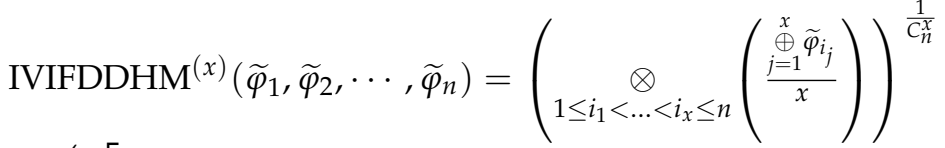

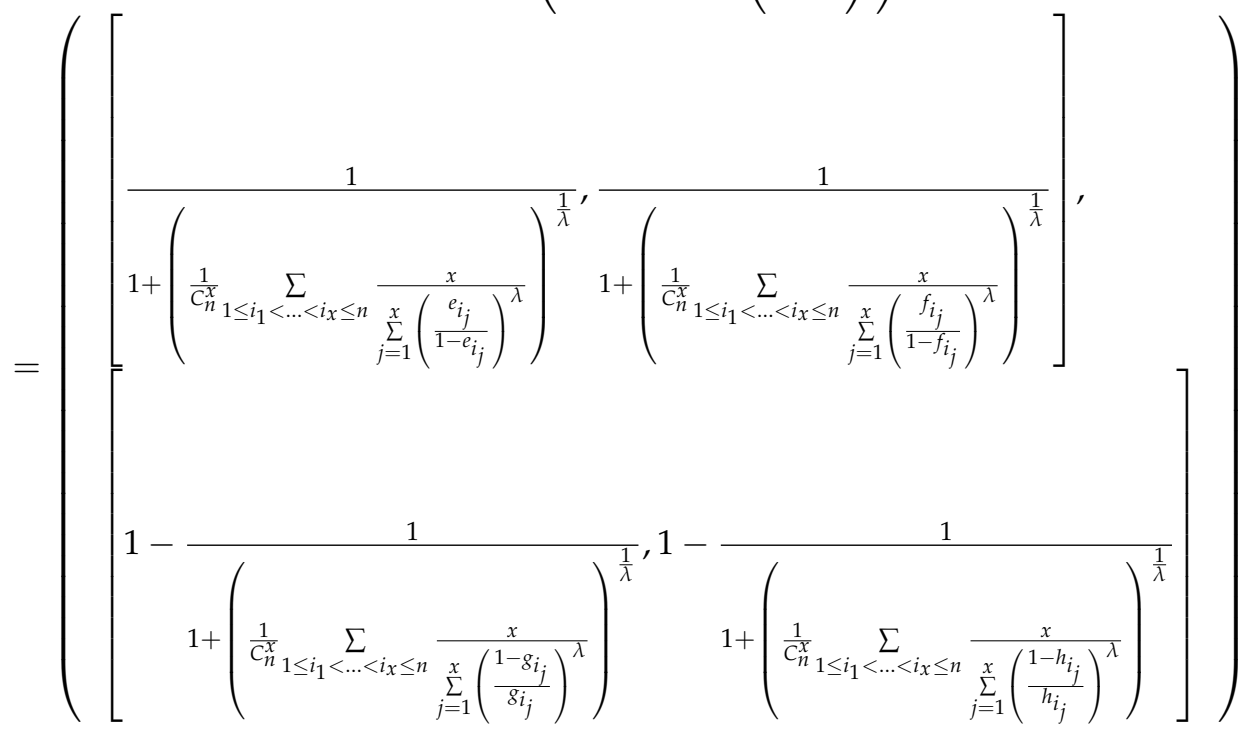




\section{Proof.}

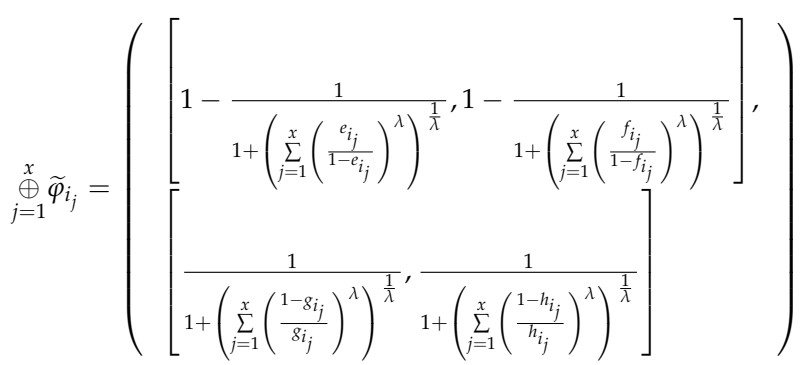

Thus,

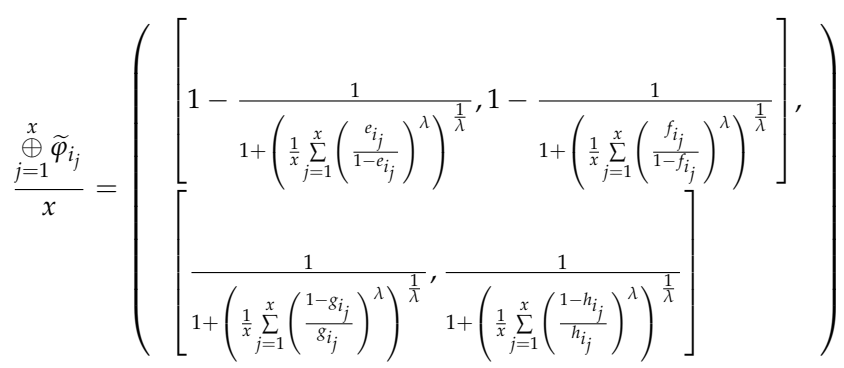

Therefore,

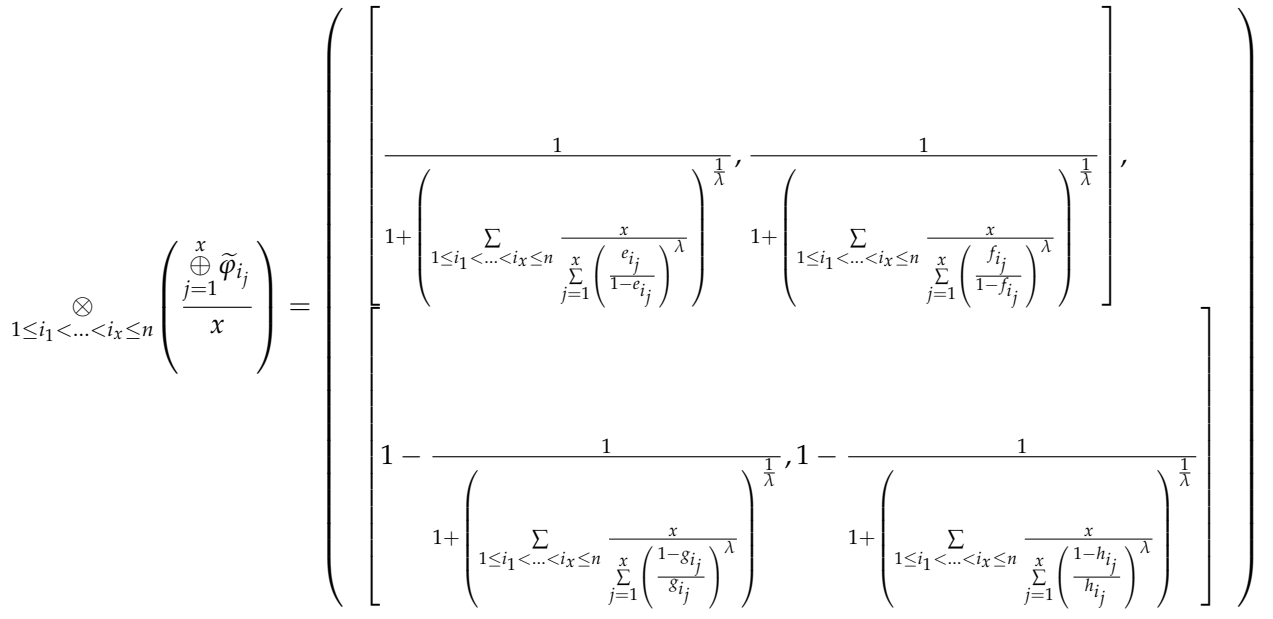

Therefore,

$$
\begin{aligned}
& \operatorname{IVIFDDHM}^{(x)}\left(\widetilde{\varphi}_{1}, \widetilde{\varphi}_{2}, \cdots, \widetilde{\varphi}_{n}\right)=\left(\underset{1 \leq i_{1}<\ldots<i_{x} \leq n}{\otimes}\left(\frac{\stackrel{x}{\oplus} \widetilde{\varphi}_{i_{j}}}{x}\right)\right)^{\frac{1}{C_{n}^{x}}}
\end{aligned}
$$

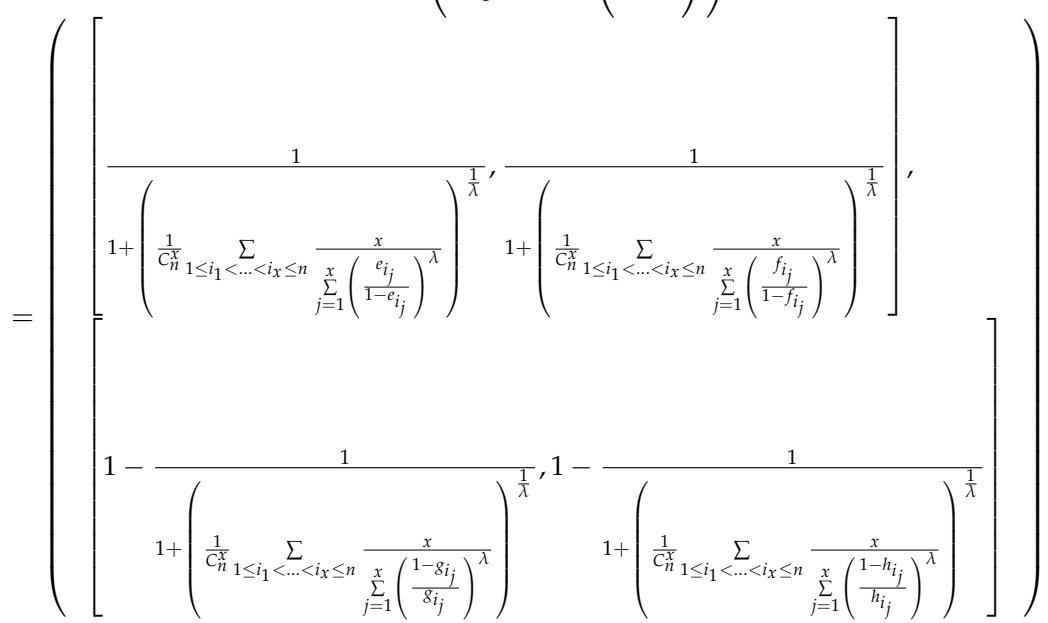

Thus, Equation (28) is right. $\square$ 
Example 3. Let $\widetilde{\varphi}_{1}=([0.2,0.4],[0.3,0.6]), \widetilde{\varphi}_{2}=([0.1,0.3],[0.2,0.5]), \widetilde{\varphi}_{3}=([0.3,0.5],[0.1,0.2])$ and $\widetilde{\varphi}_{4}=([0.1,0.4],[0.3,0.5])$ be four IVIFNs, and $x=2, \lambda=3$,

$$
\begin{gathered}
\frac{e_{i_{j}}}{1-e_{i_{j}}}=(0.2500,0.1111,0.4286,0.1111), \frac{f_{i_{j}}}{1-f_{i_{j}}}=(0.6667,0.4286,1.0000,0.6667) \\
\frac{1-g_{i_{j}}}{g_{i_{j}}}=(2.3333,4.0000,9.0000,2.3333), \frac{1-h_{i_{j}}}{h_{i_{j}}}=(0.6667,1.0000,4.0000,1.0000)
\end{gathered}
$$

Then according to Equation (28), we have

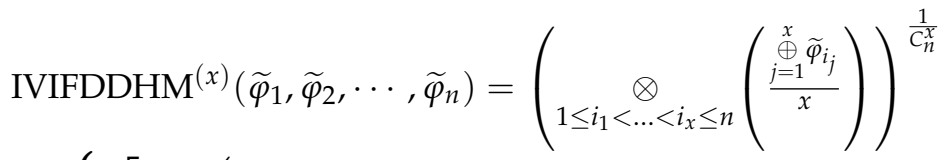

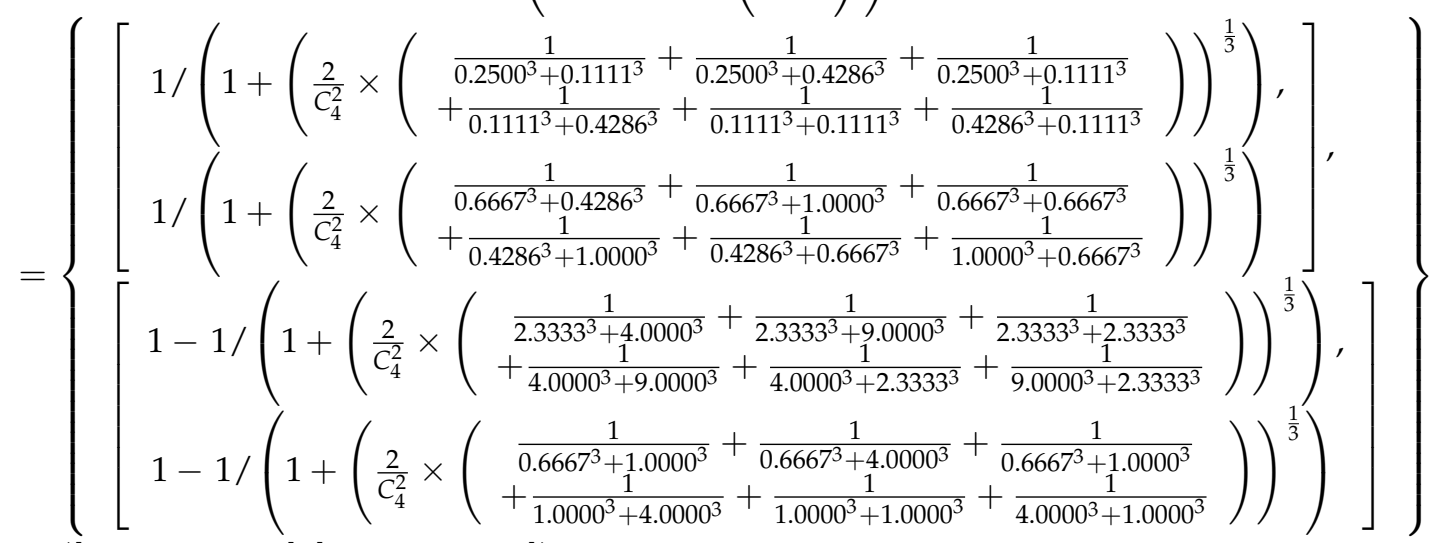

$$
\begin{aligned}
& =([0.1523,0.4052],[0.2217,0.4699])
\end{aligned}
$$

The IVIFDDHM operator has the following properties.

Property 6. (Idempotency) If $\widetilde{\varphi}_{j}=\left(\left[e_{j}, f_{j}\right],\left[g_{j}, h_{j}\right]\right)(j=1,2, \ldots, n)$ are equal, then

$$
\operatorname{IVIFDDHM}^{(x)}\left(\widetilde{\varphi}_{1}, \widetilde{\varphi}_{2}, \cdots, \widetilde{\varphi}_{n}\right)=\widetilde{\varphi}
$$

Property 7. (Monotonicity) Let $\widetilde{\varphi}_{j}=\left(\left[e_{j}, f_{j}\right],\left[g_{j}, h_{j}\right]\right)(j=1,2, \ldots, n)$ and $\widetilde{\theta}_{j}=\left(\left[r_{j}, s_{j}\right],\left[m_{j}, n_{j}\right]\right)$ $(j=1,2, \ldots, n)$ be two sets of IVIFNs. If $e_{j} \leq r_{j}, f_{j} \leq s_{j}$ and $g_{j} \geq m_{j}, h_{j} \geq n_{j}$ hold for all $j$, then

$$
\operatorname{IVIFDDHM}^{(x)}\left(\widetilde{\varphi}_{1}, \widetilde{\varphi}_{2}, \cdots, \widetilde{\varphi}_{n}\right) \leq \operatorname{IVIFDDHM}^{(x)}\left(\widetilde{\theta}_{1}, \widetilde{\theta}_{2}, \cdots, \widetilde{\theta}_{n}\right)
$$

Property 8. (Boundedness) Let $\widetilde{\varphi}_{j}=\left(\left[e_{j}, f_{j}\right],\left[g_{j}, h_{j}\right]\right)(j=1,2, \ldots, n)$ be a set of IVIFNs. If $\widetilde{\varphi}_{i}^{+}=\left(\left(\left[\max _{i}\left(e_{j}\right), \max _{i}\left(f_{j}\right)\right],\left[\min _{i}\left(g_{j}\right), \min _{i}\left(h_{j}\right)\right]\right)\right)$ and $\widetilde{\varphi}_{i}^{-}=\left(\left[\min _{i}\left(e_{j}\right), \min _{i}\left(f_{j}\right)\right]\right.$, $\left.\left[\max _{i}\left(g_{j}\right), \max _{i}\left(h_{j}\right)\right]\right)$ then

$$
\widetilde{\varphi}^{-} \leq \operatorname{IVIFDDHM}^{(x)}\left(\widetilde{\varphi}_{1}, \widetilde{\varphi}_{2}, \cdots, \widetilde{\varphi}_{n}\right) \leq \widetilde{\varphi}^{+}
$$

\subsection{The IVIFWDDHM Operator}

In practical MADM, it is important to pay attention to attribute weights; we propose the interval-valued intuitionistic weighted Dombi DHM (IVIFWDDHM) operator. 
Definition 14. Let $\widetilde{\varphi}_{j}=\left(\left[e_{j}, f_{j}\right],\left[g_{j}, h_{j}\right]\right)(j=1,2, \ldots, n)$ be a set of IVIFNs with their weight vector be $w_{i}=\left(w_{1}, w_{2}, \ldots, w_{n}\right)^{T}$, thereby satisfying $w_{i} \in[0,1]$ and $\sum_{i=1}^{n} w_{i}=1$.

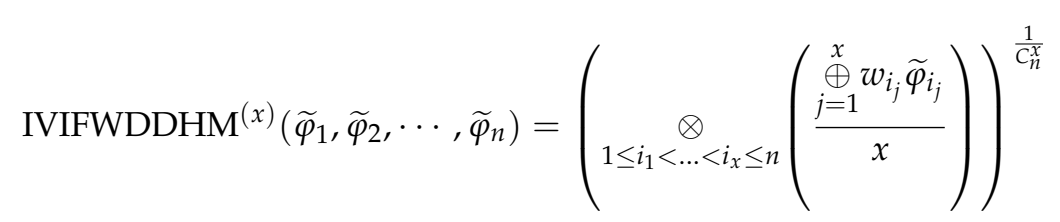

Theorem 4. Let $\widetilde{\varphi}_{j}=\left(\left[e_{j}, f_{j}\right],\left[g_{j}, h_{j}\right]\right)(j=1,2, \ldots, n)$ be a set of IVIFNs. The fused value by IVIFWDDHM operators is also an IVIFN where

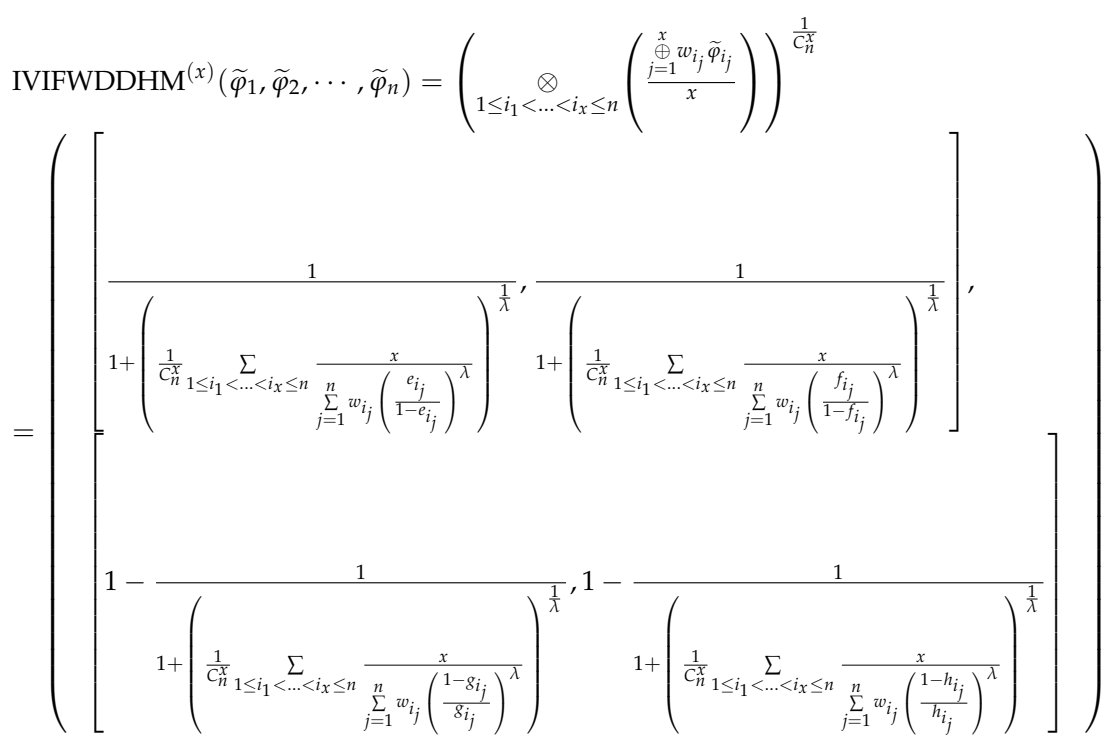

\section{Proof.}

$$
w_{i_{j}} \widetilde{\varphi}_{i_{j}}=\left(\begin{array}{l}
{\left[1-\frac{1}{\left.1+\left(w_{i_{j}}\left(\frac{e_{i_{j}}}{1-e_{i_{j}}}\right)^{\lambda}\right)^{\frac{1}{\lambda}}, 1-\frac{1}{1+\left(w_{i_{j}}\left(\frac{f_{i_{j}}}{1-f_{i_{j}}}\right)^{\lambda}\right)^{\lambda}}\right]}\right.} \\
{\left[\frac{1}{\left.1+\left(w_{i_{j}}\left(\frac{1-g_{i_{j}}}{g_{i_{j}}}\right)^{\lambda}\right)^{\frac{1}{\lambda}}, \frac{1}{1+\left(w_{i_{j}}\left(\frac{1-h_{i_{j}}}{h_{i_{j}}}\right)^{\lambda}\right)^{\frac{1}{\lambda}}}\right]}\right)}
\end{array}\right.
$$

Then,

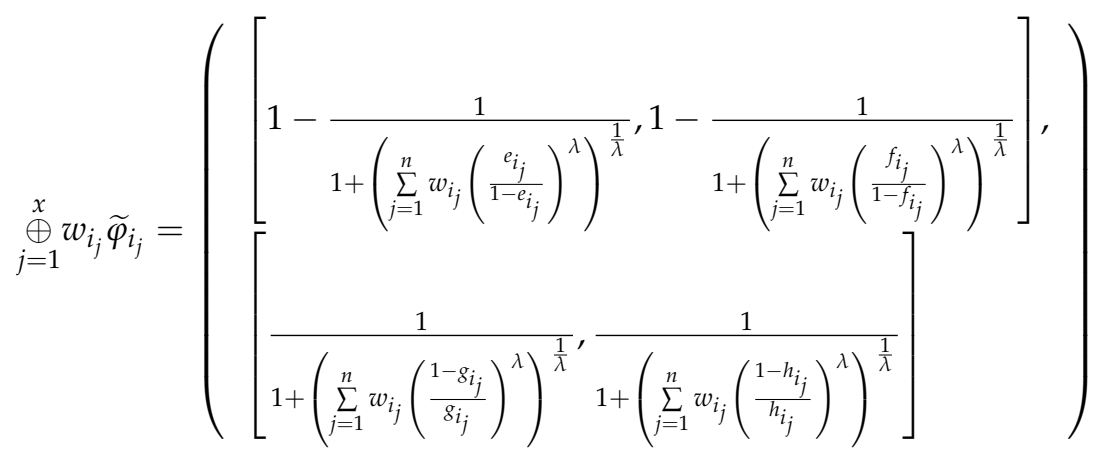


Thus,

$$
\frac{\bigoplus_{j=1}^{x} w_{i_{j}} \widetilde{\varphi}_{i_{j}}}{x}=\left(\begin{array}{l}
{\left[1-\frac{1}{1+\left(\frac{1}{x} \sum_{j=1}^{n} w_{i_{j}}\left(\frac{e_{i_{j}}}{1-e_{i_{j}}}\right)^{\lambda}\right)^{\frac{1}{\lambda}}}, 1-\frac{1}{1+\left(\frac{1}{x} \sum_{j=1}^{n} w_{i_{j}}\left(\frac{f_{i_{j}}}{1-f_{i_{j}}}\right)^{\lambda}\right)^{\frac{1}{\lambda}}}\right],} \\
\left.\frac{1}{1+\left(\frac{1}{x} \sum_{j=1}^{n} w_{i_{j}}\left(\frac{1-g_{i_{j}}}{s_{i_{j}}}\right)^{\lambda}\right)^{\frac{1}{\lambda}}}, \frac{1}{1+\left(\frac{1}{x} \sum_{j=1}^{n} w_{i_{j}}\left(\frac{1-h_{i_{j}}}{h_{i_{j}}}\right)^{\lambda}\right)^{\frac{1}{\lambda}}}\right]
\end{array}\right)
$$

\section{Therefore,}

$$
\begin{aligned}
& \underset{1 \leq i_{1}<\ldots<i i_{x} \leq n}{\otimes}\left(\frac{\substack{x \\
\oplus=1}}{x}\right)
\end{aligned}
$$

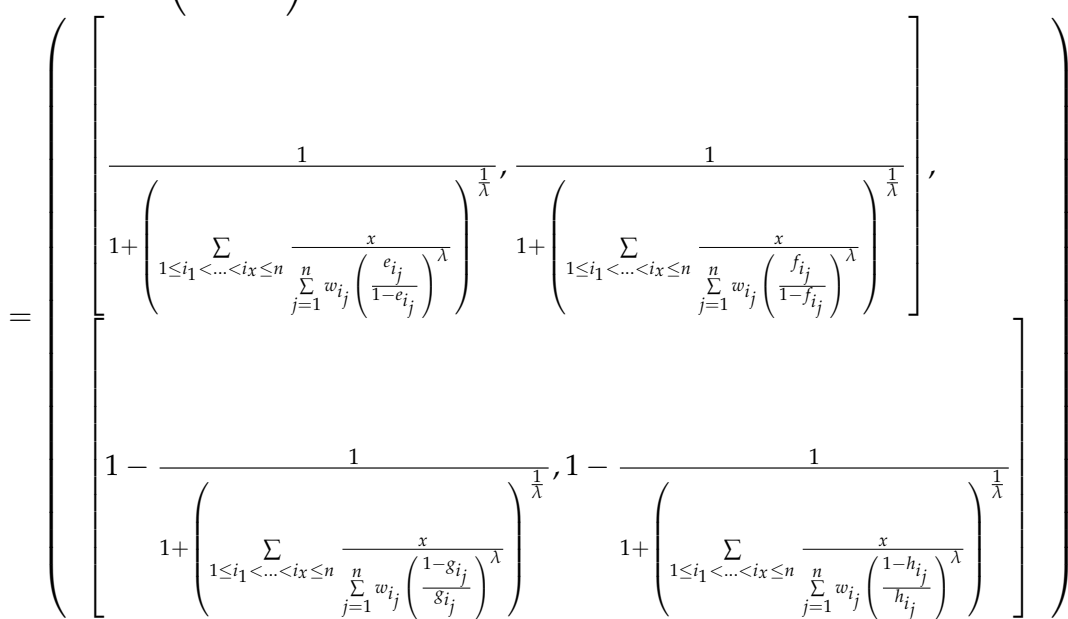

Therefore,

$$
\begin{aligned}
& \operatorname{IVIFWDDHM}^{(x)}\left(\widetilde{\varphi}_{1}, \widetilde{\varphi}_{2}, \cdots, \widetilde{\varphi}_{n}\right)=\left(\underset{1 \leq i_{1}<\ldots<i_{x} \leq n}{\otimes}\left(\frac{\substack{x \\
j=1}}{x}\right)\right)^{i_{i_{j}} \widetilde{\varphi}_{i_{j}}}
\end{aligned}
$$

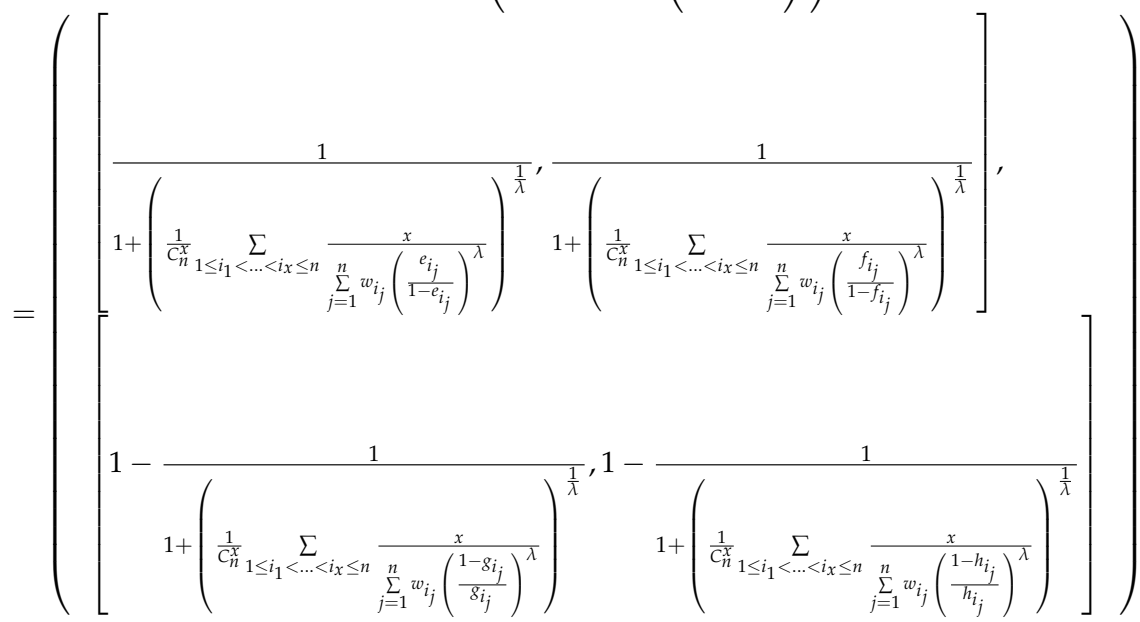

Thus, Equation (37) is right. $\square$ 
Example 4. Let $\widetilde{\varphi}_{1}=([0.2,0.4],[0.3,0.6]), \widetilde{\varphi}_{2}=([0.1,0.3],[0.2,0.5]), \widetilde{\varphi}_{3}=([0.3,0.5],[0.1,0.2])$ and $\widetilde{\varphi}_{4}=([0.1,0.4],[0.3,0.5])$ be four IVIFNs, and $x=2, \lambda=3, w=(0.4,0.1,0.2,0.3)$,

$$
\begin{gathered}
\frac{e_{i_{j}}}{1-e_{i_{j}}}=(0.2500,0.1111,0.4286,0.1111), \frac{f_{i_{j}}}{1-f_{i_{j}}}=(0.6667,0.4286,1.0000,0.6667) \\
\frac{1-g_{i_{j}}}{g_{i_{j}}}=(2.3333,4.0000,9.0000,2.3333), \frac{1-h_{i_{j}}}{h_{i_{j}}}=(0.6667,1.0000,4.0000,1.0000)
\end{gathered}
$$

Then according to Equation (37), we have

$$
\begin{aligned}
& \operatorname{IVIFWDDHM}^{(x)}\left(\widetilde{\varphi}_{1}, \widetilde{\varphi}_{2}, \cdots, \widetilde{\varphi}_{n}\right)=\left(\underset{1 \leq i_{1}<\ldots<i_{x} \leq n}{\otimes}\left(\frac{\stackrel{x}{\oplus} w_{i_{j}} \widetilde{\varphi}_{i_{j}}}{x}\right)\right)^{\frac{1}{\mathcal{C}_{n}^{x}}}
\end{aligned}
$$

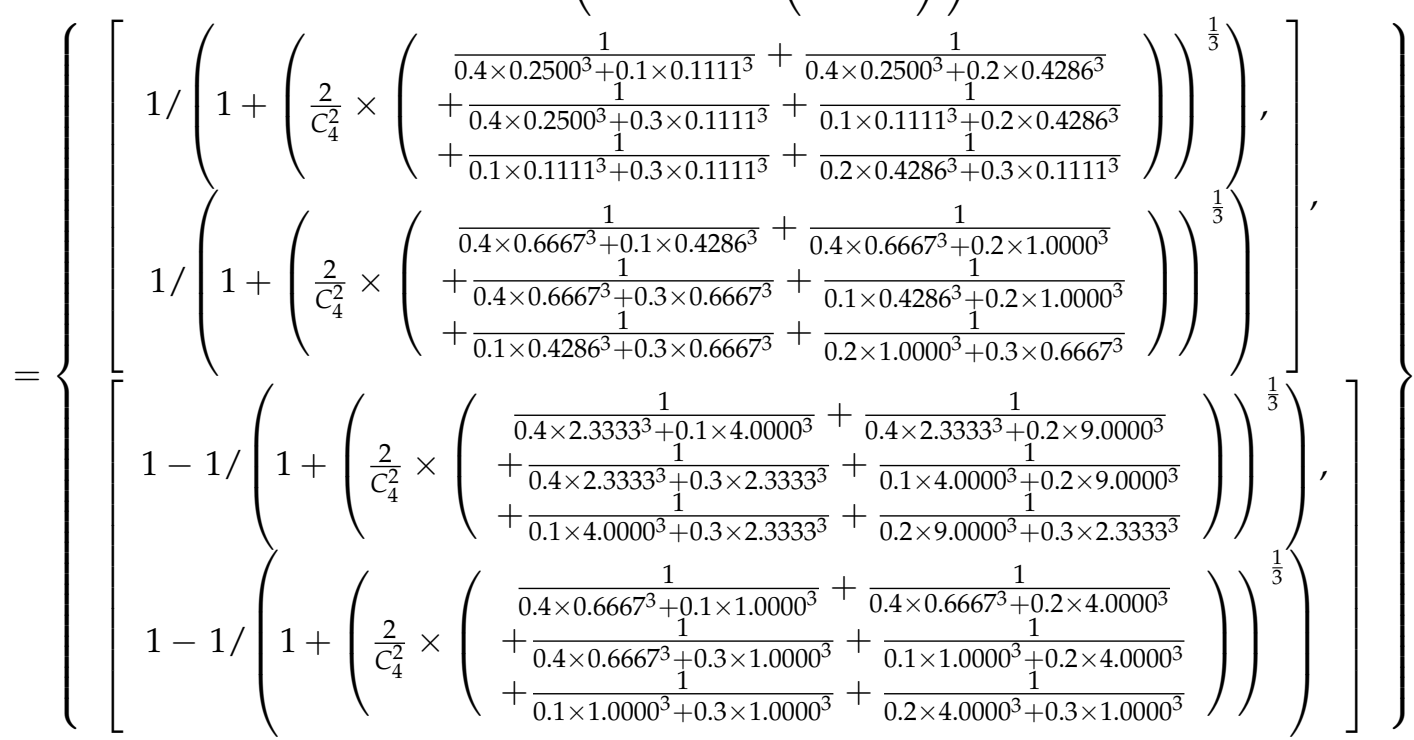

$$
\begin{aligned}
& =([0.0917,0.2962],[0.3283,0.6086])
\end{aligned}
$$

Then we give some properties of the IVIFWDDHM operator.

Property 9. (Monotonicity) Let $\widetilde{\varphi}_{j}=\left(\left[e_{j}, f_{j}\right],\left[g_{j}, h_{j}\right]\right)(j=1,2, \ldots, n)$ and $\widetilde{\theta}_{j}=\left(\left[r_{j}, s_{j}\right],\left[m_{j}, n_{j}\right]\right)$ $(j=1,2, \ldots, n)$ be two sets of IVIFNs. If $e_{j} \leq r_{j}, f_{j} \leq s_{j}$ and $g_{j} \geq m_{j}, h_{j} \geq n_{j}$ hold for all $j$, then

$$
\operatorname{IVIFWDDHM}^{(x)}\left(\widetilde{\varphi}_{1}, \widetilde{\varphi}_{2}, \cdots, \widetilde{\varphi}_{n}\right) \leq \operatorname{IVIFWDDHM}^{(x)}\left(\widetilde{\theta}_{1}, \widetilde{\theta}_{2}, \cdots, \widetilde{\theta}_{n}\right)
$$

The proof is similar to IVIFWDHM, thus, it is omitted here.

Property 10. (Boundedness) Let $\widetilde{\varphi}_{j}=\left(\left[e_{j}, f_{j}\right],\left[g_{j}, h_{j}\right]\right)(j=1,2, \ldots, n)$ be a set of IVIFNs. If $\widetilde{\varphi}_{i}^{+}=\left(\left(\left[\max _{i}\left(e_{j}\right), \max _{i}\left(f_{j}\right)\right],\left[\min _{i}\left(g_{j}\right), \min _{i}\left(h_{j}\right)\right]\right)\right)$ and $\widetilde{\varphi}_{i}^{-}=\left(\left[\min _{i}\left(e_{j}\right), \min _{i}\left(f_{j}\right)\right]\right.$, $\left.\left[\max _{i}\left(g_{j}\right), \max _{i}\left(h_{j}\right)\right]\right)$ then

$$
\widetilde{\varphi}^{-} \leq \operatorname{IVIFWDDHM}^{(x)}\left(\widetilde{\varphi}_{1}, \widetilde{\varphi}_{2}, \ldots, \widetilde{\varphi}_{n}\right) \leq \widetilde{\varphi}^{+}
$$

\section{Example and Comparison}

\subsection{Numerical Example}

With the development of the economy and society and the deepening of aging, "Senior tourism" continues to heat up. Elderly tourism has become a tourist market which cannot be ignored at present 
and in the future, and contains a tremendous potential for development. The tourism destinations are also actively involved in the development of this market. However, there are still many issues in the tourism service for the elderly tourists in the tourism destination. Although some researches on elderly tourism in China has been on the rise in recent years, many researches are conducted from the points of the consumption behavior of the elderly, the development of the elderly tourism market and the development of elderly tourism products, but the research on elderly tourism services, especially the quality of elderly tourism services, is relatively scarce. Additionally, the problems of evaluating the elderly tourism service quality in tourism destination are classical MADM problems [55-62]. Thus, we give an example to solve the MADM problems for evaluating the elderly tourism service quality in tourism destination with IVIFNs. There are five possible tourism scenic spots $A_{i}(i=1,2,3,4,5)$ to assess. The experts use the four attributes to assess the five tourism scenic spots: 1 ( $\mathrm{G}_{1}$ is the resource safety value; (2) $G_{2}$ is the infrastructure construction value; (3) $G_{3}$ is the income distribution value; (4) $\mathrm{G}_{4}$ is the promotion employment value. The five possible tourism scenic spots are to be assessed with IVIFNs (whose weighting vector $\omega=(0.4,0.1,0.3,0.2)$ ), as shown in the Table 1 .

Table 1. The IVIFN decision matrix.

\begin{tabular}{ccccc}
\hline & $\mathbf{G}_{\mathbf{1}}$ & $\mathbf{G}_{\mathbf{2}}$ & $\mathbf{G}_{3}$ & $\mathbf{G}_{\mathbf{4}}$ \\
\hline $\mathrm{A}_{1}$ & $([0.5,0.6],[0.1,0.2])$ & $([0.4,0.6],[0.2,0.4])$ & $([0.2,0.3],[0.1,0.4])$ & $([0.3,0.5],[0.1,0.3])$ \\
$\mathrm{A}_{2}$ & $([0.3,0.4],[0.2,0.5])$ & $([0.4,0.5],[0.1,0.2])$ & $([0.6,0.7],[0.2,0.3])$ & $([0.3,0.4],[0.2,0.4])$ \\
$\mathrm{A}_{3}$ & $([0.7,0.8],[0.1,0.2])$ & $([0.6,0.8],[0.1,0.2])$ & $([0.4,0.7],[0.1,0.3])$ & $([0.5,0.6],[0.1,0.4])$ \\
$\mathrm{A}_{4}$ & $([0.6,0.7],[0.1,0.3])$ & $([0.2,0.3],[0.6,0.7])$ & $([0.4,0.6],[0.2,0.4])$ & $([0.1,0.3],[0.4,0.5])$ \\
$\mathrm{A}_{5}$ & $([0.4,0.5],[0.1,0.3])$ & $([0.1,0.2],[0.5,0.7])$ & $([0.3,0.4],[0.5,0.6])$ & $([0.5,0.7],[0.1,0.2])$ \\
\hline
\end{tabular}

Then, we use the approach developed for selecting the best tourism scenic spots.

Step 1. According to IVIFNs $r_{i j}(i=1,2,3,4,5, j=1,2,3,4)$, we fuse all IVIFNs $r_{i j}$ by the IVIFWDHM (IVIFWDDHM) operator to have the IVIFNs $A_{i}(i=1,2,3,4,5)$ of the tourism scenic spots $A_{i}$. Let $x=2$, then the fused results are in Table 2 .

Table 2. The fused results of the tourism scenic spots by the IVIFWDHM (IVIFWDDHM) operator.

\begin{tabular}{ccc}
\hline & IVIFWDHM & IVIFWDDHM \\
\hline $\mathrm{A}_{1}$ & $([0.4739,0.631],[0.0767,0.2244])$ & $([0.2328,0.3929],[0.1613,0.4415])$ \\
$\mathrm{A}_{2}$ & $([0.5148,0.6181],[0.1262,0.2546])$ & $([0.2666,0.3582],[0.2507,0.4565])$ \\
$\mathrm{A}_{3}$ & $([0.6774,0.8127],[0.0639,0.1873])$ & $([0.4166,0.6356],[0.1531,0.3644])$ \\
$\mathrm{A}_{4}$ & $([0.4237,0.6055],[0.1902,0.3538])$ & $([0.1483,0.2887],[0.4442,0.5720])$ \\
$\mathrm{A}_{5}$ & $([0.4192,0.5342],[0.1182,0.3106])$ & $([0.2591,0.3607],[0.4856,0.6062])$ \\
\hline
\end{tabular}

Step 2. Using Table 2, the score values of the tourism scenic spots are in Table 3.

Table 3. The score values of tourism scenic spots.

\begin{tabular}{ccc}
\hline Alternatives & IVIFWDHM & IVIFWDDHM \\
\hline $\mathrm{A}_{1}$ & 0.4019 & 0.0115 \\
$\mathrm{~A}_{2}$ & 0.3760 & -0.0412 \\
$\mathrm{~A}_{3}$ & 0.6194 & 0.2673 \\
$\mathrm{~A}_{4}$ & 0.2426 & -0.2896 \\
$\mathrm{~A}_{5}$ & 0.2623 & -0.2360 \\
\hline
\end{tabular}

Step 3. Using Table 3, the order of tourism scenic spots is listed in Table 4. Additionally, the best tourism scenic spot is $A_{3}$. 
Table 4. The order of the tourism scenic spots.

\begin{tabular}{cc}
\hline Methods & Order \\
\hline IVIFWDHM & $A_{3}>A_{1}>A_{2}>A_{5}>A_{4}$ \\
IVIFWDDHM & $A_{3}>A_{1}>A_{2}>A_{5}>A_{4}$ \\
\hline
\end{tabular}

\subsection{Influence Analysis}

In order to depict the effects on the ordering by altering parameters of $x$ in the IVIFWDHM (IVIFWDDHM) operators, the analysis results are listed in Tables 5 and 6.

Table 5. The ordering results for the IVIFWDHM operator with different parameters.

\begin{tabular}{ccccccc}
\hline & $\mathbf{S}\left(\mathbf{A}_{\mathbf{1}}\right)$ & $\mathbf{S}\left(\mathbf{A}_{\mathbf{2}}\right)$ & $\mathbf{S}\left(\mathbf{A}_{\mathbf{3}}\right)$ & $\mathbf{S}\left(\mathbf{A}_{\mathbf{4}}\right)$ & $\mathbf{S}\left(\mathbf{A}_{5}\right)$ & Order \\
\hline$x=1$ & 0.4791 & 0.5436 & 0.6885 & 0.4243 & 0.4936 & $\mathrm{~A}_{3}>\mathrm{A}_{2}>\mathrm{A}_{5}>\mathrm{A}_{1}>\mathrm{A}_{4}$ \\
$x=2$ & 0.4019 & 0.3760 & 0.6194 & 0.2426 & 0.2623 & $\mathrm{~A}_{3}>\mathrm{A}_{1}>\mathrm{A}_{2}>\mathrm{A}_{5}>\mathrm{A}_{4}$ \\
$x=3$ & 0.3373 & 0.2970 & 0.5703 & 0.0878 & 0.0506 & $\mathrm{~A}_{3}>\mathrm{A}_{1}>\mathrm{A}_{2}>\mathrm{A}_{4}>\mathrm{A}_{5}$ \\
$x=4$ & 0.2643 & 0.2651 & 0.5438 & -0.0055 & -0.0284 & $\mathrm{~A}_{3}>\mathrm{A}_{2}>\mathrm{A}_{1}>\mathrm{A}_{4}>\mathrm{A}_{5}$ \\
\hline
\end{tabular}

Table 6. The ordering results for the IVIFWDDHM operator with different parameters.

\begin{tabular}{ccccccc}
\hline & $\mathbf{S}\left(\mathbf{A}_{\mathbf{1}}\right)$ & $\mathbf{S}\left(\mathbf{A}_{\mathbf{2}}\right)$ & $\mathbf{S}\left(\mathbf{A}_{\mathbf{3}}\right)$ & $\mathbf{S}\left(\mathbf{A}_{\mathbf{4}}\right)$ & $\mathbf{S}\left(\mathbf{A}_{5}\right)$ & Order \\
\hline$x=1$ & -0.1388 & -0.1036 & 0.1650 & -0.5660 & -0.5671 & $\mathrm{~A}_{3}>\mathrm{A}_{2}>\mathrm{A}_{1}>\mathrm{A}_{4}>\mathrm{A}_{5}$ \\
$x=2$ & 0.0115 & -0.0412 & 0.2673 & -0.2896 & -0.2360 & $\mathrm{~A}_{3}>\mathrm{A}_{1}>\mathrm{A}_{2}>\mathrm{A}_{5}>\mathrm{A}_{4}$ \\
$x=3$ & 0.0694 & 0.0206 & 0.3143 & 0.0232 & 0.0740 & $\mathrm{~A}_{3}>\mathrm{A}_{5}>\mathrm{A}_{1}>\mathrm{A}_{4}>\mathrm{A}_{2}$ \\
$x=4$ & 0.1354 & 0.1212 & 0.3661 & 0.1496 & 0.1163 & $\mathrm{~A}_{3}>\mathrm{A}_{4}>\mathrm{A}_{1}>\mathrm{A}_{2}>\mathrm{A}_{5}$ \\
\hline
\end{tabular}

\subsection{Comparative Analysis}

We compare the IVIFWDHM and IVIFWDDHM operators with the IVIFWA operator [54], IVIFWG operator [5], gray relational analysis method [8], and the correlation coefficient [63]. The results are listed in Table 7.

Table 7. The order of the tourism scenic spots.

\begin{tabular}{cc}
\hline Methods & Order \\
\hline IVIFWA operator [54] & $\mathrm{A}_{3}>\mathrm{A}_{1}>\mathrm{A}_{4}>\mathrm{A}_{2}>\mathrm{A}_{5}$ \\
IVIFWG operator [5] & $\mathrm{A}_{3}>\mathrm{A}_{1}>\mathrm{A}_{2}>\mathrm{A}_{4}>\mathrm{A}_{5}$ \\
Gray Relational Analysis Method [8] & $\mathrm{A}_{3}>\mathrm{A}_{5}>\mathrm{A}_{1}>\mathrm{A}_{2}>\mathrm{A}_{4}$ \\
Correlation Coefficient [63] & $\mathrm{A}_{3}>\mathrm{A}_{1}>\mathrm{A}_{2}>\mathrm{A}_{4}>\mathrm{A}_{5}$ \\
\hline
\end{tabular}

From above, we can get the same best tourism scenic spots and the four methods' ranking results are slightly different. However, the existing methods with IVIFNs do not consider the relationship information among the arguments. Our proposed IVIFWDHM and IVIFWDDHM operators consider the relationship among the aggregated arguments.

Additionally, $\mathrm{Xu}$ and Chen [9] defined the interval-valued intuitionistic fuzzy Bonferroni mean for aggregating the IVIFNs. However, these Bonferroni mean for aggregating the IVIFNs only consider the relationship information between two arguments and do not consider the relationship information among more than two arguments.

\section{Conclusions}

In this paper, we investigate the MADM problems with IVIFNs. Then, we utilize the HM operator and Dombi operations to design some HM operators with IVIFNs: IVIFDHM operator, IVIFWDHM operator, IVIFDDHM operator and IVIFWDDHM operator. The main characteristic of 
these operators are investigated. Then, we have used the IVIFWDHM and IVIFWDDHM operators to propose two models for MADM problems with IVIFNs. Finally, a real example for evaluating the elderly tourism service quality in the tourism destination is used to show the developed approach. In the subsequent studies, the extension and application of IVIFNs needs to be studied in many other uncertain environments and other applications.

Author Contributions: L.W., G.W., H.G., and Y.W. conceived and worked together to achieve this work, L.W. compiled the computing program by Matlab and analyzed the data, L.W. and G.W. wrote the paper. Finally, all the authors have read and approved the final manuscript.

Funding: The work was supported by the National Natural Science Foundation of China under Grant No. 71571128, the National Social Science Foundation of China under Grant No. 16CGL026 and the Construction Plan of Scientific Research Innovation Team for Colleges and Universities in Sichuan Province (15TD0004).

Conflicts of Interest: The authors declare no conflict of interest.

\section{References}

1. Atanassov, K.T. More on Intuitionistic Fuzzy-Sets. Fuzzy Sets Syst. 1989, 33, 37-45. [CrossRef]

2. Atanassov, K.T.; Gargov, G.K. Intuitionistic Fuzzy-Logic. Dokl. Bolg. Akad. Nauk. 1990, 43, 9-12.

3. Atanassov, K.; Gargov, G. Interval valued intuitionistic fuzzy-sets. Fuzzy Sets Syst. 1989, 31, $343-349$. [CrossRef]

4. Xu, Z.S. Intuitionistic fuzzy aggregation operators. IEEE Trans. Fuzzy Syst. 2007, 15, 1179-1187.

5. Xu, Z.S.; Yager, R.R. Some geometric aggregation operators based on intuitionistic fuzzy sets. Int. J. Gen. Syst. 2006, 35, 417-433. [CrossRef]

6. Xu, Z.S.; Chen, J. On Geometric Aggregation Ove Interval-Valued Intuitionistic Fuzzy Information. In Proceedings of the 4th International Conference on Fuzzy Systems and Knowledge Discovery, Haikou, China, 24-27 August 2007.

7. Wei, G.W. Some induced geometric aggregation operators with intuitionistic fuzzy information and their application to group decision making. Appl. Soft Comput. 2010, 10, 423-431. [CrossRef]

8. Wei, G.W. Gray relational analysis method for intuitionistic fuzzy multiple attribute decision making. Expert Syst. Appl. 2011, 38, 11671-11677. [CrossRef]

9. $\mathrm{Xu}, \mathrm{Z}$.S.; Chen, Q. A multi-criteria decision making procedure based on interval-valued intuitionistic fuzzy bonferroni means. J. Syst. Sci. Syst. Eng. 2011, 20, 217-228. [CrossRef]

10. Deng, X.M.; Wei, G.W.; Gao, H.; Wang, J. Models for Safety Assessment of Construction Project with Some 2-Tuple Linguistic Pythagorean Fuzzy Bonferroni Mean Operators. IEEE Access 2018, 6, 52105-52137. [CrossRef]

11. Tang, X.Y.; Huang, Y.H.; Wei, G.W. Approaches to Multiple-Attribute Decision-Making Based on Pythagorean 2-Tuple Linguistic Bonferroni Mean Operators. Algorithms 2018, 11, 5. [CrossRef]

12. Tang, X.Y.; Wei, G.W. Models for Green Supplier Selection in Green Supply Chain Management with Pythagorean 2-Tuple Linguistic Information. IEEE Access 2018, 6, 18042-18060. [CrossRef]

13. Wang, J.; Tang, X.Y.; Wei, G.W. Models for Multiple Attribute Decision-Making with Dual Generalized Single-Valued Neutrosophic Bonferroni Mean Operators. Algorithms 2018, 11, 2. [CrossRef]

14. Wang, J.; Wei, G.W.; Wei, Y. Models for Green Supplier Selection with Some 2-Tuple Linguistic Neutrosophic Number Bonferroni Mean Operators. Symmetry 2018, 10, 131. [CrossRef]

15. Wei, G.W. Picture 2-Tuple Linguistic Bonferroni Mean Operators and Their Application to Multiple Attribute Decision Making. Int. J. Fuzzy Syst. 2017, 19, 997-1010. [CrossRef]

16. Wei, G.W. Picture uncertain linguistic Bonferroni mean operators and their application to multiple attribute decision making. Kybernetes 2017, 46, 1777-1800. [CrossRef]

17. Wei, G.W.; Gao, H.; Wang, J.; Huang, Y.H. Research on Risk Evaluation of Enterprise Human Capital Investment With Interval-Valued Bipolar 2-Tuple Linguistic Information. IEEE Access 2018, 6, 35697-35712. [CrossRef]

18. Chen, T.Y. The Inclusion-Based LINMAP Method for Multiple Criteria Decision Analysis Within an Interval-Valued Atanassov's Intuitionistic Fuzzy Environment. Int. J. Inf. Technol. Decis. Mak. 2014, 13, 1325-1360. [CrossRef] 
19. Hashemi, H.; Bazargan, J.; Mousavi, S.M.; Vandani, B. An extended compromise ratio model with an application to reservoir flood control operation under an interval-valued intuitionistic fuzzy environment. Appl. Math. Model. 2014, 38, 3495-3511. [CrossRef]

20. Liu, B.S.; Chen, Y.; Shen, Y.H.; Sun, H.; Xu, X.H. A complex multi-attribute large-group decision making method based on the interval-valued intuitionistic fuzzy principal component analysis model. Soft Comput. 2014, 18, 2149-2160. [CrossRef]

21. Chen, T.Y. IVIF-PROMETHEE outranking methods for multiple criteria decision analysis based on interval-valued intuitionistic fuzzy sets. Fuzzy Optim. Decis. Mak. 2015, 14, 173-198. [CrossRef]

22. Dugenci, M. A new distance measure for interval valued intuitionistic fuzzy sets and its application to group decision making problems with incomplete weights information. Appl. Soft Comput. 2016, 41, 120-134. [CrossRef]

23. Garg, H. A new generalized improved score function of interval-valued intuitionistic fuzzy sets and applications in expert systems. Appl. Soft Comput. 2016, 38, 988-999. [CrossRef]

24. Thong, N.T.; Son, L.H. HIFCF: An effective hybrid model between picture fuzzy clustering and intuitionistic fuzzy recommender systems for medical diagnosis. Expert Syst. Appl. 2015, 42, 3682-3701. [CrossRef]

25. Son, L.H. Measuring analogousness in picture fuzzy sets: From picture distance measures to picture association measures. Fuzzy Optim. Decis. Mak. 2017, 16, 359-378. [CrossRef]

26. Son, L.H.; Thong, P.H. Some novel hybrid forecast methods based on picture fuzzy clustering for weather nowcasting from satellite image sequences. Appl. Intell. 2017, 46, 1-15. [CrossRef]

27. Wei, G.W. Some Cosine Similarity Measures for Picture Fuzzy Sets and Their Applications to Strategic Decision Making. Informatica 2017, 28, 547-564. [CrossRef]

28. Wei, G.W. Picture fuzzy aggregation operators and their application to multiple attribute decision making. J. Intell. Fuzzy Syst. 2017, 33, 713-724. [CrossRef]

29. Wei, G.W. Some Similarity Measures for Picture Fuzzy Sets and Their Applications. Iran. J. Fuzzy Syst. 2018, $15,77-89$.

30. Wei, G.W. TODIM Method for Picture Fuzzy Multiple Attribute Decision Making. Informatica 2018, 29, 555-566. [CrossRef]

31. Wei, G.W. Picture Fuzzy Hamacher Aggregation Operators and their Application to Multiple Attribute Decision Making. Fundam. Inform. 2018, 157, 271-320. [CrossRef]

32. Wei, G.W.; Alsaadi, F.E.; Hayat, T.; Alsaedi, A. Projection models for multiple attribute decision making with picture fuzzy information. Int. J. Mach. Learn. Cybern. 2018, 9, 713-719. [CrossRef]

33. Wei, G.W.; Gao, H. The Generalized Dice Similarity Measures for Picture Fuzzy Sets and Their Applications. Informatica 2018, 29, 107-124. [CrossRef]

34. Zhang, X.L.; Xu, Z.S. Extension of TOPSIS to Multiple Criteria Decision Making with Pythagorean Fuzzy Sets. Int. J. Intell. Syst. 2014, 29, 1061-1078. [CrossRef]

35. Garg, H. A Novel Correlation Coefficients between Pythagorean Fuzzy Sets and Its Applications to Decision-Making Processes. Int. J. Intell. Syst. 2016, 31, 1234-1252. [CrossRef]

36. Garg, H. New exponential operational laws and their aggregation operators for interval-valued Pythagorean fuzzy multicriteria decision-making. Int. J. Intell. Syst. 2018, 33, 653-683. [CrossRef]

37. Garg, H. A Linear Programming Method Based on an Improved Score Function for Interval-Valued Pythagorean Fuzzy Numbers and Its Application to Decision-Making. Int. J. Uncertain. Fuzziness Knowl.-Based Syst. 2018, 26, 67-80. [CrossRef]

38. Li, Z.X.; Wei, G.W.; Lu, M. Pythagorean Fuzzy Hamy Mean Operators in Multiple Attribute Group Decision Making and Their Application to Supplier Selection. Symmetry 2018, 10, 505. [CrossRef]

39. Liang, D.C.; Darko, A.P.; Xu, Z.S. Interval-valued Pythagorean fuzzy extended Bonferroni mean for dealing with heterogenous relationship among attributes. Int. J. Intell. Syst. 2018, 33, 1381-1411. [CrossRef]

40. Wang, J.; Wei, G.W.; Gao, H. Approaches to Multiple Attribute Decision Making with Interval-Valued 2-Tuple Linguistic Pythagorean Fuzzy Information. Mathematics 2018, 6, 201. [CrossRef]

41. Wei, G. Pythagorean fuzzy interaction aggregation operators and their application to multiple attribute decision making (vol. 33, pp. 2119, 2017). J. Intell. Fuzzy Syst. 2018, 34, 2817-2824. [CrossRef]

42. Wei, G.W.; Lu, M. Pythagorean Fuzzy Maclaurin Symmetric Mean Operators in Multiple Attribute Decision Making. Int. J. Intell. Syst. 2018, 33, 1043-1070. [CrossRef] 
43. Wei, G.W.; Lu, M. Pythagorean fuzzy power aggregation operators in multiple attribute decision making. Int. J. Intell. Syst. 2018, 33, 169-186. [CrossRef]

44. Wei, G.W.; Lu, M.; Tang, X.Y.; Wei, Y. Pythagorean hesitant fuzzy Hamacher aggregation operators and their application to multiple attribute decision making. Int. J. Intell. Syst. 2018, 33, 1197-1233. [CrossRef]

45. Gulistan, M.; Wahab, H.A.; Smarandache, F.; Khan, S.; Shah, S.I.A. Some Linguistic Neutrosophic Cubic Mean Operators and Entropy with Applications in a Corporation to Choose an Area Supervisor. Symmetry 2018, 10, 428. [CrossRef]

46. Liu, P.D.; You, X.L. Some linguistic neutrosophic Hamy mean operators and their application to multi-attribute group decision making. PLOS ONE 2018, 13, e0193027. [CrossRef] [PubMed]

47. Singh, S.; Garg, H. Symmetric Triangular Interval Type-2 Intuitionistic Fuzzy Sets with Their Applications in Multi Criteria Decision Making. Symmetry 2018, 10, 401. [CrossRef]

48. Hara, Y.; Uchiyama, M.; Takahasi, S.E. A refinement of various mean inequalities. J. Inequal. Appl. 1998, 2, 387-395. [CrossRef]

49. Wu, S.J.; Wang, J.; Wei, G.W.; Wei, Y. Research on Construction Engineering Project Risk Assessment with Some 2-Tuple Linguistic Neutrosophic Hamy Mean Operators. Sustainability 2018, 10, 1536. [CrossRef]

50. Dombi, J. A General-Class of Fuzzy Operators, the Demorgan Class of Fuzzy Operators and Fuzziness Measures Induced by Fuzzy Operators. Fuzzy Sets Syst. 1982, 8, 149-163. [CrossRef]

51. Khan, Q.; Liu, P.D.; Mahmood, T.; Smarandache, F.; Ullah, K. Some Interval Neutrosophic Dombi Power Bonferroni Mean Operators and Their Application in Multi-Attribute Decision-Making. Symmetry 2018, 10, 459. [CrossRef]

52. Liu, P.D.; Liu, J.L.; Chen, S.M. Some intuitionistic fuzzy Dombi Bonferroni mean operators and their application to multi-attribute group decision making. J. Oper. Res. Soc. 2018, 69, 1-24. [CrossRef]

53. Wei, G.; Wei, Y. Some single-valued neutrosophic dombi prioritized weighted aggregation operators in multiple attribute decision making. J. Intell. Fuzzy Syst. 2018, 35, 2001-2013. [CrossRef]

54. Xu, Z.S.; Yager, R.R. Dynamic intuitionistic fuzzy multi-attribute decision making. Int. J. Approx. Reason. 2008, 48, 246-262. [CrossRef]

55. Gao, H.; Lu, M.; Wei, G.W.; Wei, Y. Some Novel Pythagorean Fuzzy Interaction Aggregation Operators in Multiple Attribute Decision Making. Fundam. Inform. 2018, 159, 385-428. [CrossRef]

56. Gao, H.; Wei, G.W.; Huang, Y.H. Dual Hesitant Bipolar Fuzzy Hamacher Prioritized Aggregation Operators in Multiple Attribute Decision Making. IEEE Access 2018, 6, 11508-11522. [CrossRef]

57. Huang, Y.H.; Wei, G.W. TODIM method for Pythagorean 2-tuple linguistic multiple attribute decision making. J. Intell. Fuzzy Syst. 2018, 35, 901-915. [CrossRef]

58. Wang, J.; Wei, G.W.; Lu, M. An Extended VIKOR Method for Multiple Criteria Group Decision Making with Triangular Fuzzy Neutrosophic Numbers. Symmetry 2018, 10, 497. [CrossRef]

59. Wang, J.; Wei, G.W.; Lu, M. TODIM Method for Multiple Attribute Group Decision Making under 2-Tuple Linguistic Neutrosophic Environment. Symmetry 2018, 10, 486. [CrossRef]

60. Wei, G.W.; Gao, H.; Wei, Y. Some q-rung orthopair fuzzy Heronian mean operators in multiple attribute decision making. Int. J. Intell. Syst. 2018, 33, 1426-1458. [CrossRef]

61. Wei, G.W.; Wei, Y. Similarity measures of Pythagorean fuzzy sets based on the cosine function and their applications. Int. J. Intell. Syst. 2018, 33, 634-652. [CrossRef]

62. Wei, G.W.; Alsaadi, F.E.; Hayat, T.; Alsaedi, A. Bipolar Fuzzy Hamacher Aggregation Operators in Multiple Attribute Decision Making. Int. J. Fuzzy Syst. 2018, 20, 1-12. [CrossRef]

63. Wei, G.W.; Wang, H.J.; Lin, R. Application of correlation coefficient to interval-valued intuitionistic fuzzy multiple attribute decision-making with incomplete weight information. Knowl. Inf. Syst. 2011, 26, 337-349. [CrossRef]

(C) 2018 by the authors. Licensee MDPI, Basel, Switzerland. This article is an open access article distributed under the terms and conditions of the Creative Commons Attribution (CC BY) license (http:/ / creativecommons.org/licenses/by/4.0/). 